\title{
Welche Entwicklungszusammenhänge zwischen Sprache, Mathematik und Arbeitsgedächtnis modulieren den Einfluss sprachlicher Kompetenzen auf mathematisches Lernen im (Vor-)Schulalter?
}

\author{
Nurit Viesel-Nordmeyer $(\mathbb{D} \cdot$ Ute Ritterfeld • Wilfried Bos
}

Eingegangen: 20. Dezember 2018 / Angenommen: 2. März 2020 / Online publiziert: 16. März 2020

(C) Der/die Autor(en) 2020

Zusammenfassung Der Entwicklungszusammenhang zwischen sprachlichen und mathematischen Fähigkeiten ist hoch komplex und noch nicht gut verstanden. Deshalb wurden Kompetenzdaten von sprachlich unauffälligen Kindern aus dem Nationalen Bildungspanel (NEPS) genutzt, um interdependente Einflüsse von sprachlichen Kompetenzen (Grammatik und Wortschatz) sowie kognitiven Fähigkeiten (zentrale Exekutive, phonologische Schleife, indirekte Maße des Arbeitsgedächtnisses) und mathematischem Lernen in der Altersspanne zwischen 4 und 8 Jahren zu untersuchen $(n=354)$. Dabei zeigte sich, dass langfristig insbesondere die grammatikalischen Fähigkeiten das mathematische Lernen beeinflussen. Zudem wurde deutlich, dass Sprache innerhalb des Zusammenspiels zwischen kognitiven Fähigkeiten und mathematischen Kompetenzen unterschiedliche Funktionen einnehmen kann: So stellt sie einerseits im Vorschulalter eine Voraussetzung für die Leistungsfähigkeit einzelner mit mathematischen Kompetenzen in Zusammenhang stehender kognitiver Fähigkeiten (zentrale Exekutive, phonologische Schleife) dar. Andererseits mediiert Sprache sowohl im Vorschul- wie auch im Grundschulalter den Einfluss der kognitiven Fähigkeiten auf das mathematische Lernen. Zudem weisen die Ergeb-

\footnotetext{
Diese Arbeit nutzt Daten des Nationalen Bildungspanels (NEPS): Startkohorte Kindergarten, https://doi.org/10.5157/NEPS:SC2:8.0.1 Die Daten des NEPS wurden von 2008 bis 2013 als Teil des Rahmenprogramms zur Förderung der empirischen Bildungsforschung erhoben, welches vom Bundesministerium für Bildung und Forschung (BMBF) finanziert wurde. Seit 2014 wird NEPS vom Leibniz-Institut für Bildungsverläufe e. V. (LIfBi) an der Otto-Friedrich-Universität Bamberg in Kooperation mit einem deutschlandweiten Netzwerk weitergeführt.
}

\footnotetext{
N. Viesel-Nordmeyer $(\bowtie) \cdot$ W. Bos

Institut für Schulentwicklungsforschung (IFS), Technische Universität Dortmund (TU),

Vogelpothsweg 78, 44227 Dortmund, Deutschland

E-Mail: nurit.viesel@tu-dortmund.de

U. Ritterfeld

Fakultät Rehabilitationswissenschaften, Sprache und Kommunikation, Technische Universität

Dortmund (TU), Dortmund, Deutschland
} 
nisse darauf hin, dass sprachliche Kompetenzen insbesondere im Vorschulalter eine Voraussetzung zur Speicherung mathematischer Informationen bilden.

Schlüsselwörter Sprachkompetenzen · Mathematisches Lernen · Kognitive Fähigkeiten · Arbeitsgedächtnis

\title{
Which Developmental Relationships Between Language, Mathematics and Working Memory Modulate the Influence of Linguistic Competences On Mathematical Learning in (Pre-)school Age?
}

\begin{abstract}
The developmental relationship between linguistic and mathematical skills is highly complex and not yet sufficiently understood. We used competence data of linguistically typical developed children from the German National Educational Panel Study (NEPS) to further investigate interdependent influences of linguistic skills (grammar and vocabulary) as well as cognitive skills (central executive, phonological loop, indirect measures of working memory) and mathematical learning in the age range of 4 to 8 years $(n=354)$. Results reveal that over time especially grammatical skills impact mathematical learning. We also found evidence that language can play different roles within the interplay between cognitive skills and mathematical competences: In preschool age language represents a precondition for the performance of individual cognitive skills (central executive, phonological loop) that are closely related to mathematical learning. Language also mediates the influence of cognitive skills on mathematical learning during both, preschool and elementary school age. In addition, linguistic skills are a prerequisite for storing mathematical information particularly during preschool development.
\end{abstract}

Keywords Language skills $\cdot$ Mathematical learning $\cdot$ Cognitive skills $\cdot$ Working memory

Die bisherige Forschung zum mathematischen Kompetenzerwerb zeigt eindrücklich, dass auch sprachliche Kompetenzen den Erwerb und die Anwendung mathematischer Kompetenzen beeinflussen (u. a. Prediger et al. 2019). Gleichzeitig wird deutlich, dass die Zusammenhänge außerordentlich komplex und durch zahlreiche Interdependenzen von sprachlichen und mathematischen Lernprozessen gekennzeichnet sind. Neben einer differenzierteren qualitativen Betrachtung kommunikativer und kognitiver Funktionen der Sprache innerhalb mathematischer Lernprozesse, welche eng miteinander in Beziehung stehen (Kempert et al. 2018), scheinen insbesondere die Fokussierung auf kognitive Gedächtnisvorgänge und die Rolle der Sprache innerhalb dieser Prozesse relevant. Quantitative Untersuchungen zur kognitiven Informationsverarbeitung innerhalb sprachlicher und mathematischer Lernprozesse weisen auf einen engen Zusammenhang des kognitiven Systems des Arbeitsgedächtnisses mit Erwerb und Anwendung sprachlicher (Weinert 2010) wie auch mathematischer Kompetenzen hin (Kolkman et al. 2014). Dabei scheinen zumindest im Aufbau sprachlicher Kompetenzen bereits vorhandene sprachliche Fähigkeiten das Arbeitsgedächtnis für weitere Lernvorgänge zu entlasten (Gathercole et al. 1992). 
Auch geben Ergebnisse aus der Entwicklungs- und Neuropsychologie Hinweise darauf, dass sprachliche Kompetenzen sowohl für den vorschulischen Erwerb Zahlen verarbeitender Hirnfunktionen (von Aster 2013) wie auch für die Speicherung mathematischer Inhalte (Lorenz 2012) eine Rolle spielen. Zusätzlich legen Ergebnisse neuerer Untersuchungen aus dem Bereich der quantitativen Forschung zum Zusammenhang aller drei Komponenten - Sprache, Mathematik und Arbeitsgedächtnis nahe, dass Sprache im Vorschulalter ebenso eine vermittelnde Rolle für die Einflüsse der Leistungsfähigkeit des Arbeitsgedächtnisses auf mathematische Kompetenzen einnimmt (Kyttälä et al. 2013; Röhm et al. 2017). Eine Integration dieser Ergebnisse in ein Entwicklungsmodell steht jedoch noch aus. Zum einen fehlt es an längsschnittlichen Beobachtungen und zum anderen an Untersuchungen, welche die Wirkrichtungen von Zusammenhängen beschriebener Interdependenzen im Zusammenspiel sprachlicher und mathematischer Kompetenzen unter dem Blickwinkel dieser kognitiven Funktionen von Sprache abbilden können. Die Nutzung von Daten der Startkohorte 2 des Nationalen Bildungspanels scheint geeignet, einen Beitrag zur Schließung dieser Forschungslücke zu leisten. Aufgrund des eingesetzten Erhebungsdesigns und der vorhandenen Messungen sprachlicher Kompetenzen (rezeptiver Wortschatz, Grammatikverständnis), mathematischer Kompetenzen (Arithmetik, Geometrie, Textaufgaben, Zahl-Größen-Verständnis) und kognitiver Fähigkeiten (verbales Arbeitsgedächtnis, zentrale Exekutive, kognitive Grundfähigkeiten) im Altersbereich zwischen Kindergarten und Grundschule ist es möglich, bestehende Beziehungsgefüge, welche sich zwischen dem Einfluss sprachlicher Kompetenzen auf mathematisches Lernen abspielen, näher zu beleuchten. Zudem unterstützt das quantitative Design die Notwendigkeit, allgemeingültige Aussagen zu generieren.

\section{Theoretischer Hintergrund und Forschungsstand}

\subsection{Die kommunikative und die kognitive Funktion von Sprache in mathematischen Lernprozessen}

Sprache spielt für Lernen eine bedeutsame Rolle, wobei eine kommunikative von einer kognitiven Funktion unterschieden werden kann (Kempert et al. 2018; Sfard und Kieran 2008). Beide Funktionen sind beim mathematischen Lernen eng miteinander verwoben (ebd.). Die kommunikative Funktion dient der Verständigung über den Gegenstand Mathematik während die kognitive Funktion für den Erkenntnisgewinn wichtig ist. Die kommunikative Funktion der Sprache ist in der Vermittlung mathematischer Inhalte in mündlicher und/oder schriftlicher Form und durch die Verwendung spezifischer ,sprachlicher Register“ (Halliday 2004) mathematischen Lernens offensichtlich. Bereits hier kommt auch die kognitive Funktion zum Tragen. Diese spielt beim Erschaffen eines Verständnisses der kommunizierten (mathematischen) Begriffe und Zeichen aufgrund des engen Zusammenhangs von Sprache und Denken (Beyer und Gerlach 2018) eine besondere Rolle. Die besondere Rolle behält die kognitive Funktion der Sprache bei der weiteren Auseinandersetzung mit den vermittelnden Inhalten mathematischen Lernens bei. Gleichzeitig ist die kommunikative Funktion der Sprache für den Erkenntnisprozess höchst relevant. Dies 
zeigt sich bspw. durch vertiefende Nachfragen zum Verständnis einzelner Begriffe oder Formulierungen der dargebotenen mathematischen Inhalte. Die Produktion solcher Nachfragen setzt wiederum kognitive Funktionen der Sprache voraus (Maier und Schweiger 2008). Untersuchungen zur Rolle des häuslichen Lernumfelds für die Ausbildung mathematischer Kompetenzen weisen auf die Bedeutung des beschriebenen Zusammenspiels bereits im Kontext vorschulischen mathematischen Lernens hin (u.a. Hoeft et al. 2015). Im Schulalter wird für das Zusammenspiel der kognitiven und kommunikativen Funktion der Sprache beim mathematischen Lernen die Beherrschung komplexer „sprachlicher Register“ (Halliday 2004) vorausgesetzt, welche einen erfolgreichen Erwerb mathematischen Lernens im schulischen Kontext bedingen. Für die von der Alltagssprache abzugrenzenden Register „Bildungssprache“ und „Fachsprache“ sind sowohl ein spezifischer Wortschatz als auch komplexere grammatikalische Strukturen charakteristisch (u. a. Paetsch 2016).

\subsection{Die Bedeutung sprachlicher Kompetenzen für das Zahlenverständnis}

Mathematischen Fähigkeiten im Schulalter, wie rechnerischem Denken (Kopfrechnen), liegen Fähigkeiten der abstrakten Zahlenraumvorstellung (,,mentaler Zahlenstrahl“, vgl. von Aster 2013) zugrunde. Diese beginnen sich in der frühen Kindheit zusammen mit der Sprache und dem Arbeitsgedächtnis zu entwickeln (ebd.). Mathematische Schwächen im Grundschulbereich sind überwiegend auf eine defizitäre Entwicklung des Zahl-Größen-Verständnisses zurückzuführen, welches der Vorstellung des abstrakten Zahlenraums vorausgeht (Krajewski 2014). Im Grundschulbereich scheinen diejenigen mathematischen Teilbereiche, die mentale Repräsentationsformen voraussetzen (bspw. „Zahlenraum“, „Sachrechnen“ vgl. DEMAT $1+$; Krajewski et al. 2002), stark sprachlich determiniert (vgl. SOKKE; Heinze et al. 2007). An Entwicklungsmodellen unterschiedlicher Fachdisziplinen kann verdeutlicht werden, dass der Sprache bereits für die Entwicklung dieses tiefen Zahlenverständnisses im Vorschulalter die Rolle eines fundamentalen Antriebs zukommt. Von Aster (2013) postuliert ein sogenanntes Vier-Stufenmodell der Entwicklung zahlenverarbeitender Hirnfunktionen, das die herausragende Rolle sprachlicher Kompetenzen insbesondere im Rahmen des (frühen) Vorschulalters verdeutlicht. Ausgehend von einem grundlegenden Verständnis der groben Mengenunterscheidung (Stufe 1), mit welchem Kinder bereits geboren werden, wird mit der sich entwickelnden Sprachkompetenz auch die Fähigkeit zum sprachlichen Symbolisieren einer Anzahl durch Zahlworte erworben (Stufe 2). Die sprachliche Symbolisierungsfähigkeit und die darauffolgend erworbene mathematische Symbolisierungsfähigkeit von Zahlen (Stufe 3) bilden schließlich die Grundlage zum Erwerb einer inneren zahlenräumlichen Vorstellung (,,mentaler Zahlenstrahl“; Stufe 4). Im Unterschied zu von Aster (2013) nimmt Krajewski (2014) in ihrem Entwicklungsmodell der Zahl-GrößenVerknüpfung zunächst eine parallele Entwicklung des Mengen- und Zahlenverständnisses innerhalb der ersten zwei Entwicklungsebenen an. Im Einklang mit von Aster (2013) kommt den sprachlichen Kompetenzen bereits früh eine gesonderte Rolle für die Entwicklung mathematischer Basiskompetenzen zu. Krajewski (2014) postuliert, dass die Sprache für den Erwerb von Zahlwörtern ohne Größenbezug bereits mit Einsetzen der 1. Ebene (Basisfertigkeiten) im Alter von etwa 2 Jahren eine Katalysa- 
torrolle einnimmt. Diese Rolle behält die Sprache bis zur vollständigen Verknüpfung von Zahlwörtern/Ziffern mit Größenrelationen (Ebene 3: „tiefes Zahlenverständnis“, mit etwa 5 bis 6 Jahren) bei. Auf Ebene 2 (einfaches Zahlverständnis mit 4 bis 5 Jahren) und Ebene 3 (tiefes Zahlverständnis) gewinnen aufgrund der Notwendigkeit des Verständnisses numerischer Mengenrelationen zusätzlich visuell-räumliche Repräsentationsformen, neben den sprachlichen Repräsentationsformen, an Bedeutung.

\subsection{Die Bedeutung des Arbeitsgedächtnisses für mathematisches Lernen}

Neben der Sprache spielt auch das in die Informationsverarbeitung des Lernens eingebundene kognitive System des Arbeitsgedächtnisses für die Entwicklung des abstrakten Zahlenverständnisses eine wichtige Rolle (Kaufman und von Aster 2012). In Anlehnung an Baddeley (2000) kann das Arbeitsgedächtnis in die übergeordnete zentrale Exekutive sowie eine sprachliche und visuell-räumliche Speicherkomponente unterteilt werden. Zwar besteht Konsens darüber, dass die Leistungsfähigkeit dieses kognitiven Systems unmittelbar mit der Entwicklung und Anwendung mathematischen Wissens zusammenhängt (Grube und Seitz-Stein 2012), dennoch bleibt unklar, welche Komponenten relevant sind. So zeigen Untersuchungen unter Einschluss aller drei Komponenten des Arbeitsgedächtnisses für den frühen Altersbereich zwischen 4 und 6 Jahren insbesondere einen Einfluss der zentralen Exekutive (Ehlert 2007; Diaz-Barriga Yanez et al. 2016) auf mathematisches Lernen. Diese Komponente des Arbeitsgedächtnisses scheint speziell für neu erworbene und somit noch relativ ungeübte mathematische Prozeduren wichtig (Schröder und Ritterfeld 2014). Im späteren Vorschulalter (5 bis 6 Jahre) weisen Ergebnisse von Studien, welche zwischen verbalem und visuellem Arbeitsgedächtnis unterscheiden, auf einen zunehmenden Einfluss des visuell-räumlichen Notizblocks auf vorschulische Mengen-Zahlen-Kompetenzen hin (Cornu et al. 2017). Dagegen scheint der phonologischen Schleife eine wesentliche Rolle für mathematische Leistungen im frühen Schulalter zuzukommen (Ehlert 2007). Das bedeutet zum einen, dass mit zunehmendem Alter der Kinder und möglicherweise auch damit einhergehenden ansteigendem Automatisierungsgrad von mathematischen Prozessen, unterschiedliche Komponenten des Arbeitsgedächtnisses eine besondere Rolle spielen (ebd.). Zum anderen wird aber auch eine hohe Komplexität im Zusammenspiel einzelner Arbeitsgedächtniskomponenten innerhalb einzelner Entwicklungszeiträume deutlich. Das wiederholte Auftreten der Bedeutung der zentralen Exekutive neben beiden Speicherkomponenten zum Lösen von Textaufgaben sowie arithmetischen Aufgabenstellungen im weiteren Schulalter (Zheng et al. 2011) unterstützt die Kenntnis weiterer Funktionen der zentralen Exekutive im Kontext späteren mathematischen Lernens. Diese können u.a. durch das Abrufen von Zählstrategien aus dem Langzeitgedächtnis sowie der Koordination zwischen sprachlichen und numerischen Informationen erklärt werden (Diaz-Barriga Yanez et al. 2016), was bereits auf ein Zusammenspiel aller drei Komponenten hinweist. 


\subsection{Zusammenhänge zwischen sprachlichen Kompetenzen und Arbeitsgedächtnisleistungen}

Mittlerweile ist der Einfluss der phonologischen Schleife auf sprachliches Lernen gut dokumentiert (z.B. Götze et al. 2000). Dabei zeigte sich für den Wortschatz, dass nicht nur der erfolgreiche Erwerb und Aufbau sprachlicher Kompetenzen vom Ausmaß vorhandener Gedächtnisleistungen abhängig ist. Umgekehrt erhöhen die bereits verfügbaren sprachlichen Fähigkeiten die weitere Leistungsfähigkeit nachfolgender kognitiver Verarbeitungsprozesse, vermutlich durch eine Entlastung der Kapazität dieses kognitiven Systems (vgl. Hasselhorn und Gold 2006). So stellten Gathercole et al. (1992) im Rahmen ihrer Längsschnittstudie zum Wortschatzerwerb fest, dass sich das kausale Beziehungsgefüge zwischen Wortschatzerwerb und phonologischem Arbeitsgedächtnis altersabhängig verändert. Zwar zeigen die Ergebnisse für den Altersbereich zwischen vier und fünf Jahren den vermuteten und inzwischen durch zahlreiche Studien belegten Einfluss des phonologischen Arbeitsgedächtnisses auf den Erwerb lexikalischen Wissens. Im Alter von 6 Jahren jedoch kann nun umgekehrt das lexikalische Wissen als Prädiktor der Leistungsfähigkeit der phonologischen Schleife identifiziert werden. Dagegen ist für diese Altersstufe ein direkter Einfluss der phonologischen Schleife auf die Wortschatzleistungen mit 6 Jahren kaum noch auffindbar. Es ist deshalb anzunehmen, dass die phonologische Schleife vor allem beim Neuerwerb von Wörtern in der frühen Erwerbsphase eine ausschlaggebende Rolle spielt. Mit fortlaufendem Aufbau des Lexikons dreht sich der Wirkzusammenhang um, weil dann die Leistungsfähigkeit der kapazitätsbegrenzten phonologischen Schleife durch das bereits bestehende sprachliche Wissen erhöht wird (vgl. Weinert 2010). Allerdings ist dieser Vorgang nicht an das Alter der Kinder gebunden, denn beim Fremdsprachenerwerb nimmt die phonologische Schleife auch noch bei älteren Kindern einen deutlichen Einfluss auf den Worterwerb (Wen 2012). Das interdependente Zusammenspiel von Wortschatz und Arbeitsgedächtnis lässt sich auch auf die mathematische Kompetenzentwicklung übertragen, wie etwa beim Aufbau der Zahlenraumvorstellung (vgl. 1.2). Die hierfür nötigen verbalen Denkprozesse setzen sprachliche Fertigkeiten voraus (vgl. von Aster 2013). Der Aufbau des sprachlichen Wissens ermöglicht und beschleunigt die notwendigen Denkprozesse bei dem Erwerb und der Anwendung von weiterem Wissen (z. B. Weinert 2010). Inwieweit sich diese interdependenten Prozesse auch beim Zusammenhang zwischen phonologischen und grammatischen Kompetenzen mit dem Arbeitsgedächtnis finden, ist bisher noch nicht ausreichend untersucht worden. Unklar ist zudem, ob diese wechselseitigen Prozesse auch mit den beiden anderen Komponenten des Arbeitsgedächtnisses bestehen. Einzelne Studien weisen zumindest darauf hin, dass beim Erwerb grammatikalischer Strukturen auch die zentrale Exekutive eine Rolle spielt (Wen 2012), wenngleich hier die Rolle der phonologischen Schleife noch nicht eindeutig geklärt ist. So finden sich Belege eines direkten Zusammenhangs der phonologischen Schleife mit dem Erwerb grammatikalischer Kompetenzen (Götze et al. 2000), aber auch Hinweise auf indirekte Einflüsse, die über den Wortschatz mediiert werden (Adams und Gathercole 1996). 


\subsection{Zusammenhänge zwischen sprachlichen Kompetenzen, mathematischen Kompetenzen und Arbeitsgedächtnis}

Wie eingangs erwähnt, liefern Untersuchungen im Vorschulalter mittlerweile erste Anhaltspunkte über das Zusammenspiel aller drei Indikatoren - Sprache, Mathematik und Arbeitsgedächtnis. Demnach scheinen sprachliche Kompetenzen zusätzlich eine vermittelnde Rolle für einzelne Effekte des Arbeitsgedächtnisses auf mathematisches Lernen einzunehmen. So konnten Röhm und Kollegen (2017) feststellen, dass neben einem direkten Zusammenhang zwischen der zentralen Exekutive und den mathematischen Basiskompetenzen auch indirekte Zusammenhänge der phonologischen Schleife über die sprachlichen Kompetenzen (hier: Morphologie, Syntax) mit den mathematischen Basiskompetenzen bestehen. Kytällä und Kollegen (2013) fanden ebenfalls einen indirekten Zusammenhang der phonologischen Schleife sowohl mit bildlich als auch mündlich gemessenem numerischen Wissen über lexikalische und grammatikalische Maße. Jedoch lassen die beiden Untersuchungen aufgrund der verwendeten querschnittlichen Studiendesigns keine tatsächlichen Rückschlüsse auf die Richtung der berichteten Zusammenhänge zu. Ergebnisse einer Längsschnittstudie an Grundschulen (Hoese 2017) können dem Anspruch kausaler Aussagen zumindest für das Zusammenspiel zwischen mathematischen Kompetenzen (hier: Geometrie, Arithmetik, Sachrechnen) und grundlegenden kognitiven Fähigkeiten (gemessen durch den KFT; Abou-Koura et al. 2005; Heller und Perleth 2000) gerecht werden. Wie bereits für das Zusammenspiel zwischen Arbeitsgedächtnis und Wortschatz belegt (Gathercole et al. 1992), finden sich in der Studie von Hoese (2017) wechselseitige Zusammenhänge zwischen den gemessenen kognitiven Fähigkeiten und den erhobenen mathematischen Kompetenzen. Diese Zusammenhänge stellen sich aber, anders als beim Wortschatzaufbau (Gathercole et al. 1992), im längsschnittlichen Verlauf zwischen allen Erhebungswellen bidirektional dar. Die Befunde zum längsschnittlichen Zusammenspiel kognitiver Fähigkeiten und mathematischer Kompetenzen (Hoese 2017) können als Hinweis auf kausale Zusammenhänge zwischen den drei hier interessierenden Domänen - Sprache, Mathematik und Arbeitsgedächtnis - interpretiert werden. Der nonverbale Teil des KFT lässt die Abbildung indirekter Messungen der beiden Arbeitsgedächtnismaße zentrale Exekutive und visuell-räumlicher Notizblock vermuten ${ }^{1}$ (vgl. auch Engel de Abreu et al. 2010). Gleichzeitig bildet der verbale Teil des KFT auch (rezeptive) Sprachmaße ab. Dennoch erlauben die zur Verfügung stehenden Informationen - aufgrund der verwendeten Operationalisierung im Kontext des Forschungsinteresses der beschriebenen Untersuchung - keine differenzierte Abgrenzung aller drei hier interessierenden Komponenten, welche zur Aufklärung vorhandener Interdependenzen im Zusammenspiel von Sprache, Mathematik und Arbeitsgedächtnis notwendig wären. Die Annahme, dass kognitive Fähigkeiten für den Wissensaufbau notwendig sind

\footnotetext{
${ }_{1}^{1}$ Die Lösung herkömmlich eingesetzter Matrizen-Tests zur nonverbalen Messung der fluiden Intelligenz benötigt Gedächtnisleistungen, die auch bei der Lösung von Spannenaufgaben zur Messung des Arbeitsgedächtnisses angesprochen werden. Einerseits wird eine visuelle Speicherkomponente zur Aufrechterhaltung der Informationen benötigt. Zur weiteren Verarbeitung sind zudem zentral-exekutive Arbeitsgedächtnisfunktionen erforderlich (Engel de Abreu et al. 2010).
} 


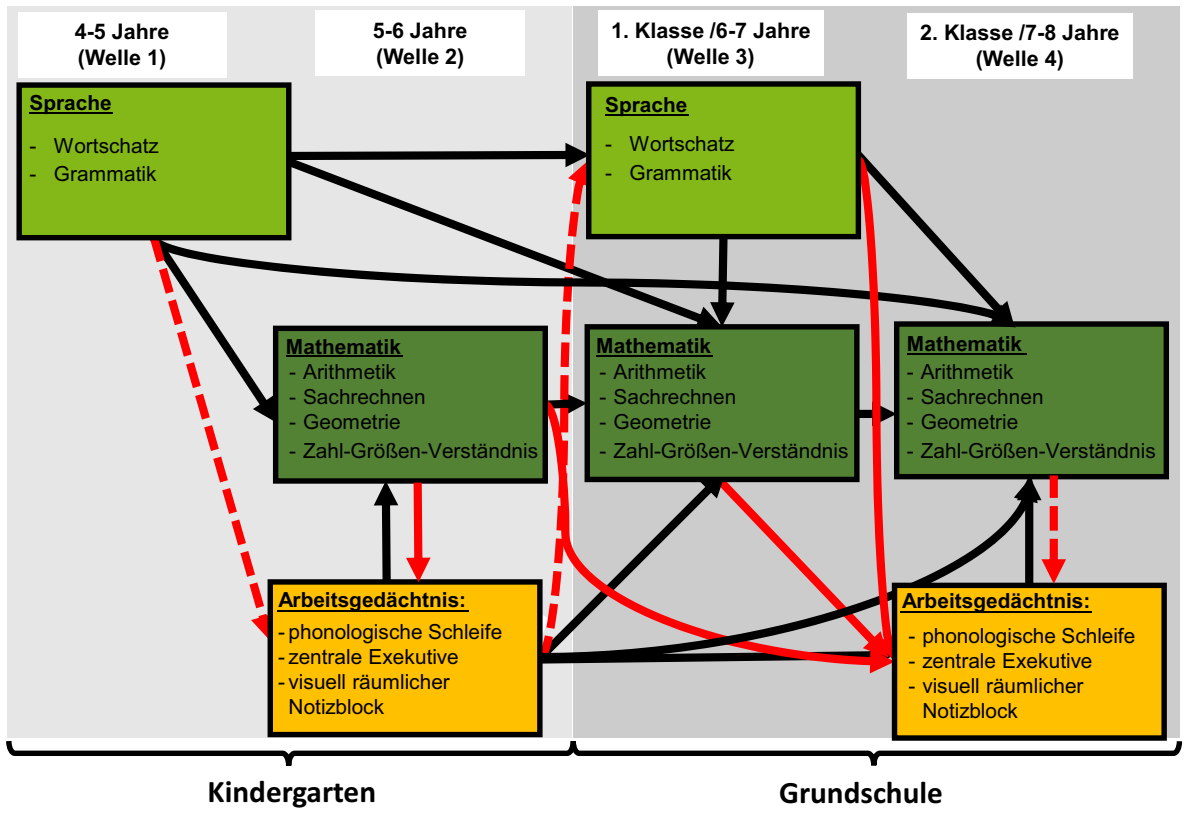

Abb. 1 Rahmenmodell angenommener Zusammenhänge zwischen Sprache, Mathematik und Arbeitsgedächtnis der bei NEPS (SC 2) verfügbaren Variablen vom Kindergarten (4-5 Jahre) bis in die zweite Klassenstufe (7-8 Jahre). Anmerkungen. Bereits bekannte Zusammenhänge des Forschungsfelds werden schwarz dargestellt, unbekannte Zusammenhänge rot, teilweise bekannte rot-gestrichelt

und umgekehrt, bereits bestehendes Wissen die kognitive Leistungsfähigkeit erhöht, scheint durch die beschriebenen Befunde von Hoese (2017) jedoch zumindest für den mathematischen Kompetenzerwerb im Schulalter bestätigt.

\section{Ziel der vorliegenden Untersuchung}

Durch die Darlegung des bestehenden Forschungsstandes konnte eine hohe Komplexität des Einflusses sprachlicher Kompetenzen auf mathematisches Lernen und unter Beachtung des in diese Lernprozesse eingebundene kognitiven Systems des Arbeitsgedächtnisses identifiziert werden. Dabei erweist sich die Frage nach der Gesamtheit dieses komplexen Beziehungsgefüges hinsichtlich bestehender Interdependenzen innerhalb der Entwicklungsspanne mathematischen Lernens vom Kindergarten bis in die Grundschule noch als unbeantwortet (vgl. Abb. 1). Aufgrund der Notwendigkeit allgemeingültige Aussagen bezüglich dieses komplexen Beziehungsgefüges zu erreichen, verspricht die Durchführung quantitativer Analysen mittels eines aussagekräftigen Paneldatensatzes einen Erkenntnisgewinn. Dabei sollen in Anlehnung an den bereits vorhandenen Forschungsstand verfügbare Kompetenzdaten der Startkohorte 2 des Nationalen Bildungspanels (NEPS) in ein Rahmenmodell des beschriebenen Beziehungsgefüges integriert werden. Die beschriebene Integration hebt die Möglichkeit der Verwendung dieses längsschnittlichen Datensatzes als 
vielversprechend hervor (vgl. Abb. 1). Zudem erlauben vorhandene Informationen zu Migrationshintergrund, sozioökonomischem Status sowie der zuhause gesprochenen Sprache Beeinflussungen dieses komplexen Beziehungsgefüges zu kontrollieren. Das übergeordnete Ziel der vorliegenden Untersuchung ist folglich, zugrundeliegende vermittelnde Effekte im Zusammenspiel sprachlicher und mathematischer Kompetenzentwicklung unter Verwendung von Daten der Startkohorte 2 des Nationalen Bildungspanels (NEPS) näher zu betrachten. Einen ersten Schritt stellt die (1) Identifizierung direkter Einflüsse sprachlicher Kompetenzen auf mathematisches Lernen im Vorschul- und Grundschulalter dar. Aufbauend auf Erkenntnissen vorheriger Studien - welche kognitive Verarbeitungsprozesse des Arbeitsgedächtnisses mit einbeziehen - (vgl. 1.5), ist daraufhin den Fragen nachzugehen (2a) welche wechselseitigen Einflüsse im Aufbau sprachlicher Kompetenzen zwischen der Leistungsfähigkeit des Arbeitsgedächtnisses und der Ausprägung sprachlicher Kompetenzen selbst bestehen bzw. (2b) welche wechselseitigen Einflüsse im Aufbau mathematischer Kompetenzen zwischen der Leistungsfähigkeit des Arbeitsgedächtnisses und der Ausprägung mathematischer Kompetenzen selbst vorliegen. In Anlehnung an die Untersuchungen von Gathercole et al. (1992) zum Wortschatzaufbau im Vorschulalter sowie den Ergebnissen der Studie von Hoese (2017) zum mathematischen Lernen im Grundschulalter werden wechselseitige Einflüsse zwischen sprachlichen (Wortschatz und Grammatik) wie auch mathematischen und direkt bzw. indirekt gemessenen Arbeitsgedächtniskomponenten (vgl. 32) angenommen. Dabei soll speziell ein eventuelles Fortbestehen dieser bidirektionalen Einflüsse vom Vorschul- bis ins Grundschulalter hinterfragt werden.

Aufbauend auf modelltheoretischen Hintergründen zur Entwicklung eines tieferen Zahlenverständnisses (Krajewski 2014; von Aster 2013) sowie des engen Zusammenhangs der Entwicklung sprachlicher Kompetenzen und kognitiver Fähigkeiten (z. B. Kempert et al. 2018) soll darüber hinaus untersucht werden, (3a) welche Rolle sprachliche Kompetenzen innerhalb der angenommenen wechselseitigen Entwicklungszusammenhänge zwischen mathematischen Kompetenzen und der Leistungsfähigkeit des Arbeitsgedächtnisses spielen und (3b) welche Rolle die angenommenen wechselseitigen Entwicklungszusammenhänge (zwischen sprachlichen Kompetenzen und der Leistungsfähigkeit des Arbeitsgedächtnisses sowie zwischen mathematischen Kompetenzen und der Leistungsfähigkeit des Arbeitsgedächtnisses) für den direkten Einfluss von Sprache auf mathematisches Lernen im Vor- und Grundschulalter einnehmen.

\section{Methode}

\subsection{Stichprobe}

Grundlage der verwendeten Stichprobe bilden die Kompetenzdaten der Gruppe 3 (,Längsschnitt“) der Startkohorte 2 (SC2) des Nationalen Bildungspanels (NEPS) (Blossfeld et al. 2011) des Messzeitraums vom Vorschulalter (4-5 Jahre) bis zum Ende der zweiten Klassenstufe (7-8 Jahre) ( $n=504)$. Ergänzend wurden Daten zur Abbildung von Schülermerkmalen hinzugezogen, welche im Rahmen von Befragun- 
gen der Eltern und pädagogischen Fachkräfte sowie der Leitungen der Kindergärten bzw. Schulen erhoben wurden.

Im Rahmen eines organisatorisch begründeten Testabbruchs ${ }^{2}$ wurden zur weiteren Vergleichbarkeit der Daten 78 Personen ausgeschlossen $(n=426)$. Nach Kontrolle der Subgruppe, bei welcher es zum Testabbruch kam, kann der Testabbruch auf ein rein systematisches Problem zurückgeführt werden. Zudem wurden nur Daten derjenigen Kinder in die Analysen aufgenommen, bei denen sichergestellt werden konnte, dass keine die Testungen beeinträchtigende Behinderung wie bspw. Höroder Sehschwäche vorlag $(n=371)$. Daten von Kindern mit auffälligem kognitivem Profil $(<-1,5 S D)$ wurden exkludiert $(n=354)$. Für das Vorliegen einer Behinderung wie auch für die Verteilung des kognitiven Profils ist nicht anzunehmen, dass diese durch weitere Drittvariablen vorhersagbar sind. Demnach wurde von einer Imputation zum Erhalt der Ausgangsstichprobengröße abgesehen (Garson 2015). Der verwendete Datensatz der vorliegenden Analysen weist damit eine Stichprobengröße von $n=354$ (weiblich $=50 \%$ ) auf.

\subsection{Instrumente}

Kompetenzmessungen wurden in altersangepasster Form in Einzel- (Vorschulalter) bzw. Gruppensettings (Grundschulalter) unter Verwendung der im Folgenden aufgeführten Instrumente durchgeführt. Abhängig von der Testanfälligkeit für Memoryeffekte wurden die den Analysen zugrunde liegenden Daten unter Nutzung des Anker-Item bzw. Anker-Gruppen-Design verlinkt (Fischer et al. 2016). Die berichtete interne Konsistenz unter Verwendung von Cronbachs Alpha $(\alpha)$ bezieht sich auf die ausgewählte Stichprobe $(n=354)^{3}$.

Sprache. Sprachliche Kompetenzen wurden sowohl auf Wort- (Wortschatz) wie auch auf Satzebene (Grammatik) anhand international anschlussfähiger Verfahren rezeptiv erfasst: Eine adaptierte Form des Peabody Picture Vocabulary Tests (PPVT; Dunn und Dunn 2007) (Welle 1: $\alpha=0,85$; Welle 3: $\alpha=0,79$ ) wurde zur Erhebung von Wortschatzkompetenzen eingesetzt. Gemessene grammatische Kompetenzen (Welle 1: $\alpha=0,82$; Welle 3: $\alpha=0,80$ ) basieren auf einer gekürzten Version der deutschen Übersetzung des Test for Reception of Grammar von Bishop (1989) (TROG-D; Fox 2006). Zudem wurde die sprachliche Vorläuferfähigkeit der phonologischen Bewusstheit im Rahmen von drei Untertests mit 5-6 Jahren erhoben. Eingesetzte Untertests bilden das Oneset-Reim-Synthetisieren aus dem TPB (Fricke und Schäfer 2008; $\alpha=0,93$ ), die Reime aus dem BISC (Jansen et al. 2002; $\alpha=0,66$ ) sowie die

\footnotetext{
2 Im Rahmen von Kompetenztestungen des Grammatikverständnisses innerhalb der 3. Welle (1. Klasse) kam es bei $12,9 \%$ der Testteilnehmenden aus organisatorischen Gründen zu einem frühzeitigen Testabbruch (vgl. https://www.neps-data.de/Portals/0/NEPS/Datenzentrum/Forschungsdaten/SC2/3-0-0/NEPS_ SC2_Competences_W3_de.pdf). Die bei NEPS dokumentierten Prozentangaben des Testabbruchs gehen auf die Gesamtstichprobe zurück, welche nach der Datenauffrischung zum Grundschuleintritt (Gruppe 1, $n=6733$ ) vorlag. Der Ausschluss der Fälle mit frühzeitigem Testabbruch vermindert, abzüglich der fehlenden Angaben $(n=16)$, die hier verwendete Stichprobe der Gruppe 3 („Längsschnitt“) der Startkohorte 2 um $12,7 \%(n=62)$.

3 Eine genaue Dokumentation der bei NEPS eingesetzten Kompetenztests findet sich unter: https://www. neps-data.de/de-de/datenzentrum/datenunddokumentation/startkohortekindergarten/dokumentation.aspx.
} 
Identifikation von Phonemen aus dem MÜSC (Mannhaupt 2006; $\alpha=0,80$ ). Alle drei Skalen der Untertests wurden für die Analysen gleichwertig zusammengefasst. Zur Abbildung schriftsprachlicher Kompetenzen wurde in der zweiten Klassenstufe eine Kurzversion des ELFE-Tests 1-6 (Lenhard und Schneider 2006) eingesetzt, welche als Maß der frühen Lesekompetenz das Textverständnis $(\alpha=0,88)$ abbildet.

Mathematik. Mathematische Kompetenzen (Welle 2: $\alpha=0,79$; Welle 3: $\alpha=0,75$; Welle 4: $\alpha=0,79)$ wurden in Anlehnung an die Konzeption des ,Mathematical Literacy" (wie u.a. bei PISA definiert) sowie die Bildungsstandards der Mathematik mittels eines eigens durch NEPS konzipierten Tests erhoben (vgl. Neumann et al. 2013). Gemessene Kompetenzen umfassen dabei Teilbereiche der Arithmetik, des Sachrechnens und der Geometrie.

Kognitive Fähigkeiten. Im Rahmen der Erhebungen zur phonologischen Informationsverarbeitung wurden zwei Subkomponenten des Arbeitsgedächtnisses abgebildet (Berendes et al. 2013). Damit liegen im Vorschulbereich Daten der phonologischen Schleife (,Zahlenspanne vorwärts“, vgl. Kaufman Assessment Battery for Children; K-ABC; Melchers und Preuß 2009; $\alpha=0,74)$ sowie der zentralen Exekutive (,Zahlenspanne rückwärts“, vgl. Hamburg-Wechsler-Intelligenztest für Kinder III; HAWK-III; Tewes et al. 1999; $\alpha=0,50)$ vor. Die gering ausgeprägte interne Konsistenz der beiden Tests ist auf eine niedrige Anzahl an Items zurückzuführen (z. B. Bortz und Döring 2016), welche besonders in die Reliabilitätsanalyse der zentralen Exekutive eingeflossen ist. Als Maße der kognitiven Grundfähigkeiten wurden zwei Teilkomponenten im Vor- und Grundschulalter nonverbal erfasst. Die Wahrnehmungsgeschwindigkeit (Welle 2: $\alpha=0,82$; Welle 4: $\alpha=0,74$ ) wurde im Rahmen eines „Bilder-Zeichen-Test“ (NEPS-BZT) als Weiterentwicklung des ,Digit-Symbol-Tests (DST)“ aus der Wechsler-Familie (Lang et al. 2007) erhoben. Durch Einsatz eines klassischen Reasoning-Tests als Matrizentest konnten Maße des schlussfolgernden Denkens (Welle 2: $\alpha=0,73$; Welle 4: $\alpha=0,70$ ) abgebildet werden. Beide Skalen der kognitiven Grundfähigkeiten wurden für die vorliegenden Analysen zusammengefasst.

Die bei NEPS eingesetzten nonverbalen Testungen der kognitiven Grundfähigkeiten ermöglichten es, diese als zusätzliche indirekte Maße des Arbeitsgedächtnisses (zentrale Exekutive, visuelle Speicherkomponente) im Vor- und Grundschulalter zu interpretieren (z. B. Engel de Abreu et al. 2010). Die im Ergebnisteil noch als allgemeine kognitive Grundfähigkeiten bezeichneten Fähigkeiten wurden im Diskussionsteil dann spezifisch als indirektes Maß der ,zentralen Exekutive“ benannt. Diese indirekten Maße beinhalten zudem die Abbildung einer visuellen Speicherkomponente (ebd.).

Die verwendeten Maße für die mathematischen Kompetenzen standen als längsschnittlich verankerte Personenparameter (WLEs) zur Verfügung, was eine direkte Abbildung der im Fokus stehenden mathematischen Entwicklung ermöglichte. Angaben zu sprachlichen Kompetenzen und kognitiven Fähigkeiten gehen hingegen auf Skalenmittelwerte zurück, welche nur als Summenwerte mit unterschiedlicher Itemanzahl vorlagen. Aufgrund des Forschungsinteresses, die einzelnen Werte sprachlicher Kompetenzen und kognitiver Fähigkeiten im Zusammenhang mit der mathematischen Entwicklung abbilden zu können, wurden die einzelnen Summenwerte standardisiert. 


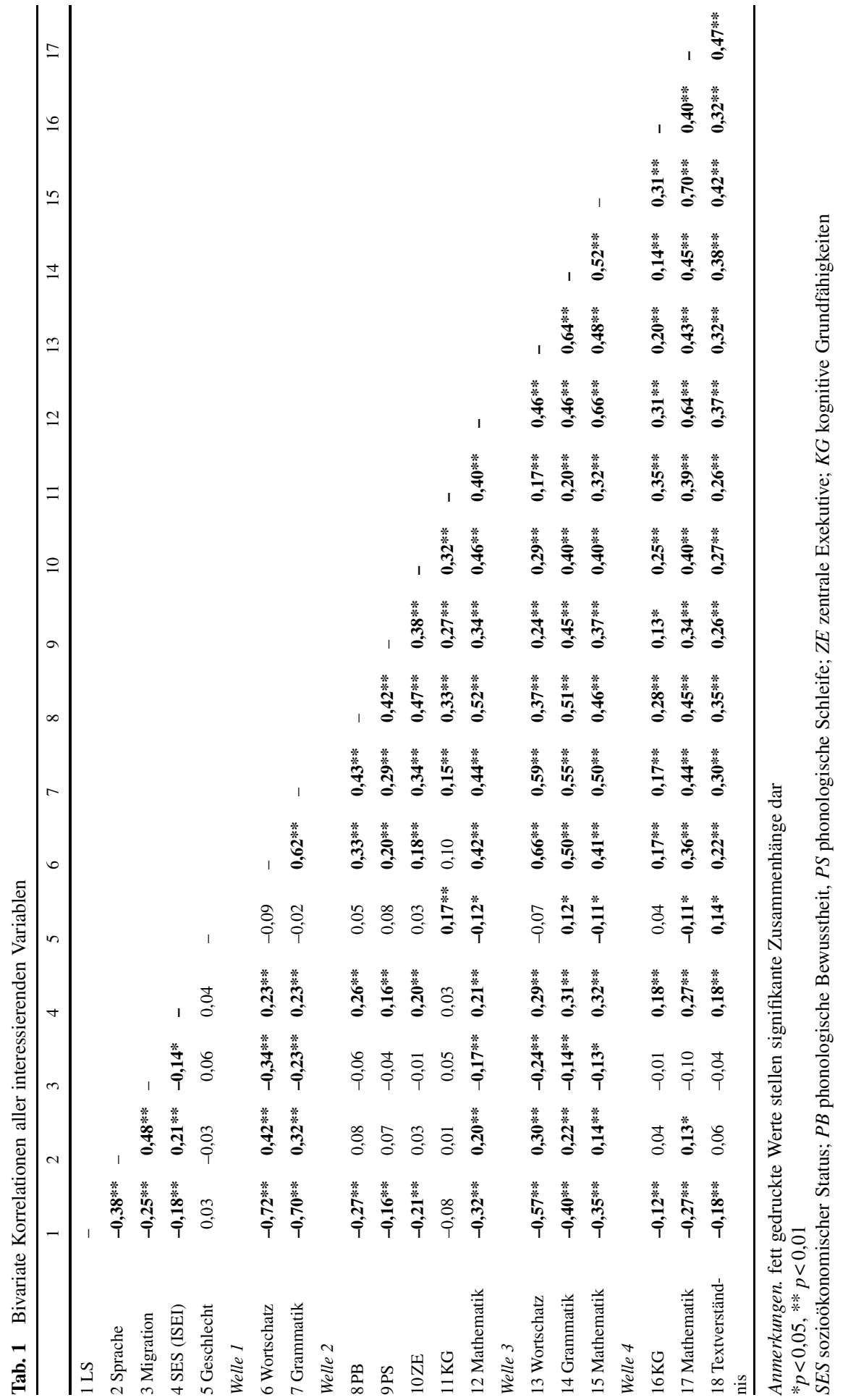


Kovariaten. Angaben zur sprachlichen Lernschwäche $(0=$ nein $/ 1=$ ja $)$ wurden unter gleichwertiger Verwendung sprachlicher Kompetenzdaten (Wortschatz und Grammatik) des ersten Messzeitpunkts (Welle 1) im Vorschulalter gebildet $(<-1 S D)$. Individuelle Merkmale wie Geschlecht ( $1=$ männlich/2 = weiblich), Migration $(\leq 3,5$ Generationen: $1=$ vorhanden $/ 0=$ nicht vorhanden) sozioökonomischer Status (ISEI08; Ganzeboom 2010) und Deutsch als überwiegend zuhause gesprochene Sprache $(0=$ nein/1 = ja) wurden Angaben der Elternbefragungen entnommen.

\subsection{Auswertung}

Zur Beantwortung der vorliegenden Fragestellungen wurden mit Hilfe des Datenverarbeitungsprogramms Mplus 5.21 (Muthen und Muthen 2009) Pfadanalysen durchgeführt. Vertiefende Mediationsanalysen wurden zur Aufdeckung indirekter Effekte berechnet. Für die Analysen wurde robuste Schätzverfahren wie Maximum-Likelihood mit robusten Standardfehlern (MLR) bzw. Bootstrapping genutzt (Christ und Schlüter 2012). Die Zusammenhänge der einzelnen Kompetenzen wurden hinsichtlich möglicher Indikatoren sprachlicher Beeinflussung durch weitere Drittvariablen kontrolliert. Somit konnte ausgeschlossen werden, dass Zusammenhänge innerhalb der Kompetenzentwicklung durch sprachliche Defizite aufgrund einer bestehenden sprachlichen Lernschwäche, der zuhause gesprochenen Sprache, dem Vorliegen eines Migrationshintergrunds oder eines niedrigen sozioökonomischen Hintergrunds verzerrt werden können. Da Einflüsse der Kontrollvariablen auf die gemessenen Kompetenzen nicht den Fokus der vorliegenden Untersuchung darstellen, wurden sie in das Modell nicht integriert. Zur Kontrolle direkter vs. indirekter Effekte wurden im Rahmen der vertiefenden Analysen zusätzliche sprachliche Variablen (phonologische Bewusstheit, Textverständnis) zur Kontrolle eingesetzt, die nicht im Gesamtmodell enthalten sind. Vorangehende deskriptive Analysen erfolgten mit SPSS 25. Für die kognitiven Grundfähigkeiten, die hier ergänzende, indirekte Maße des Arbeitsgedächtnisses bilden (vgl. Diskussion), werden im Ergebnisteil die genuinen Bezeichnungen (kognitive Grundfähigkeiten) verwendet.

\section{Ergebnisse}

Tab. 1 stellt die Zusammenhänge interessierender Variablen aller vier Messzeitpunkte mittels bivariater Korrelationen dar $(p<0,05)$. Erwartungsgemäß zeigen sich hohe Interkorrelationen der einzelnen Messungen derselben Konstrukte $(r \geq 0,55$, $p<0,01)$. Zwischen den Indikatoren sprachlicher Kompetenzen (phonologische Bewusstheit, Wortschatz, Grammatik, Textverständnis) bestehen mittlere bis hohe Zusammenhänge $(r \geq 0,20, p<0,01)$. Diese korrelieren sowohl mit mathematischen Kompetenzen $(r \geq 0,34, p<0,01)$ wie auch mit kognitiven Fähigkeiten (kognitive Grundfähigkeiten, phonologische Schleife, zentrale Exekutive) $(r \geq 0,15, p<0,01)$. Eine Ausnahme bildet der Zusammenhang zwischen dem vorschulischen Wortschatz und den kognitiven Grundfähigkeiten dieser Altersstufe $(r=0,10, p>0,05)$. Zudem hängen kognitive Grundfähigkeiten und Arbeitsgedächtnis bedeutend mit mathematischen Kompetenzen zusammen $(r \geq 0,34, p<0,01)$. Die aufgrund ihrer Rolle als 
mögliche Risikofaktoren sprachlicher Defizite eingesetzten Kontrollvariablen zeigen negative (Vorliegen einer/s sprachlichen Lernschwäche, Migrationshintergrunds; $r \leq-0,13, p<0,01$ ) bzw. positive (Deutsch als überwiegend zuhause gesprochenen Sprache, sozioökonomischer Status; $r \geq 0,14, p<0,01)$ Korrelationen mit sprachlichen und mathematischen Kompetenzen.

\subsection{Einflüsse sprachlicher Kompetenzen auf mathematisches Lernen}

Anhand des in Abb. 2 dargestellten Pfadmodells zeigt sich, dass sprachliche Kompetenzen die Entwicklung mathematischen Lernens grundlegend beeinflussen. So sagt insbesondere die Grammatik im Vorschulalter (4-5 Jahre) mathematische Leistungen im Vorschul- (5-6 Jahre: 0,33***) und Schulalter (Klasse 1: 0,19***; Klasse 2: $0,12 *)$ vorher. Dagegen scheinen frühe Wortschatzkenntnisse $(0,23 * * *)$ vorwiegend auf den Erwerb früher mathematischer Kompetenzen Einfluss zu nehmen. Innerhalb der ersten Klassenstufe hängen Sprachkenntnisse auf Wort- $\left(0,11^{*}\right)$ und auf Satzebene $\left(0,16^{* *}\right)$ mit den mathematischen Leistungen zusammen.

\subsection{Mediierte Effekte sprachlicher Kompetenzen auf mathematische Kompetenzen}

Das Grammatikverständnisses im Vorschulalter (4-5 Jahre) - welches mathematische Leistungen im Vorschul- (5-6 Jahre) und frühen Schulalter (6-7 Jahre; 1. Klasse) direkt beeinflusst (vgl. 4.1) - wirkt lediglich indirekt auf mathematische Kompetenzen der zweiten Klassenstufe ein. Mediationen bestehen einerseits über die vorangehenden mathematischen Kompetenzen selbst (Mathematik Welle 2 (5-6 Jahre): $B=0,07, S E(B)=0,02, p<0,001,95 \%$, KI für B [0,03, 0,12]; Mathematik Welle 3 (6-7 Jahre): $B=0,08, S E(B)=0,02, p<0,001,95 \%$, KI für B [0,04, 0,13]; Mathematik Welle 2 (5-6 Jahre) über Mathematik Welle 3 (6-7 Jahre): $B=0,05, S E(B)=0,02$, $p<0,001,95 \%$, KI für B $[0,01,0,09])$. Andererseits werden gering ausgeprägte Effekte über sprachliche Kompetenzen (Grammatik Welle 3 (6-7 Jahre) über mathematische Kompetenzen Welle 3 (6-7 Jahre): $B=0,05, S E(B)=0,01, p<0,001,95 \%$, KI für B $[0,03,0,07])$ wie auch über kognitive Fähigkeiten (zentrale Exekutive Welle 2 (5-6 Jahre) über mathematische Kompetenzen Welle 2 (5-6 Jahre): $B=0,05$, $S E(B)=0,01, p<0,002,95 \%$, KI für B $[0,01,0,07])$ mediiert. Vergleichbare Mediationen zeigen sich auch, zusätzlich zu dem direkten Einfluss (vgl. 4.1) des Grammatikverständnisses im Vorschulalter (4-5 Jahre), auf die mathematischen Kompetenzen des ersten Schuljahres (6-7 Jahre). Diese werden über die vorschulischen mathematischen Kompetenzen $(B=0,14, S E(B)=0,03, p<0,001,95 \%$, KI für B [0,10, $0,20])$, die Grammatikleistungen der ersten Klassenstufe $(B=0,04, S E(B)=0,02$, $p<0,007,95 \%$, KI für B $[0,02,0,08])$ wie auch über Einflüsse kognitiver Fähigkeiten im Vorschulalter (phonologische Schleife: $B=0,03, S E(B)=0,02, p<0,063$, $95 \%$, KI für B $[0,03,0,06]$; zentrale Exekutive über mathematische Kompetenzen Welle 2: $B=0,06, S E(B)=0,02, p<0,001,95 \%$, KI für B $[0,03,0,09])$ mediiert. Dieses Muster bestätigt sich nicht für Leistungen des Grammatikverständnisses der ersten Klassenstufe auf die Mathematikleistungen des kommenden Schuljahres. Hier fungieren einzig mathematische Kompetenzen der ersten Klassenstufe $(B=0,23$, 

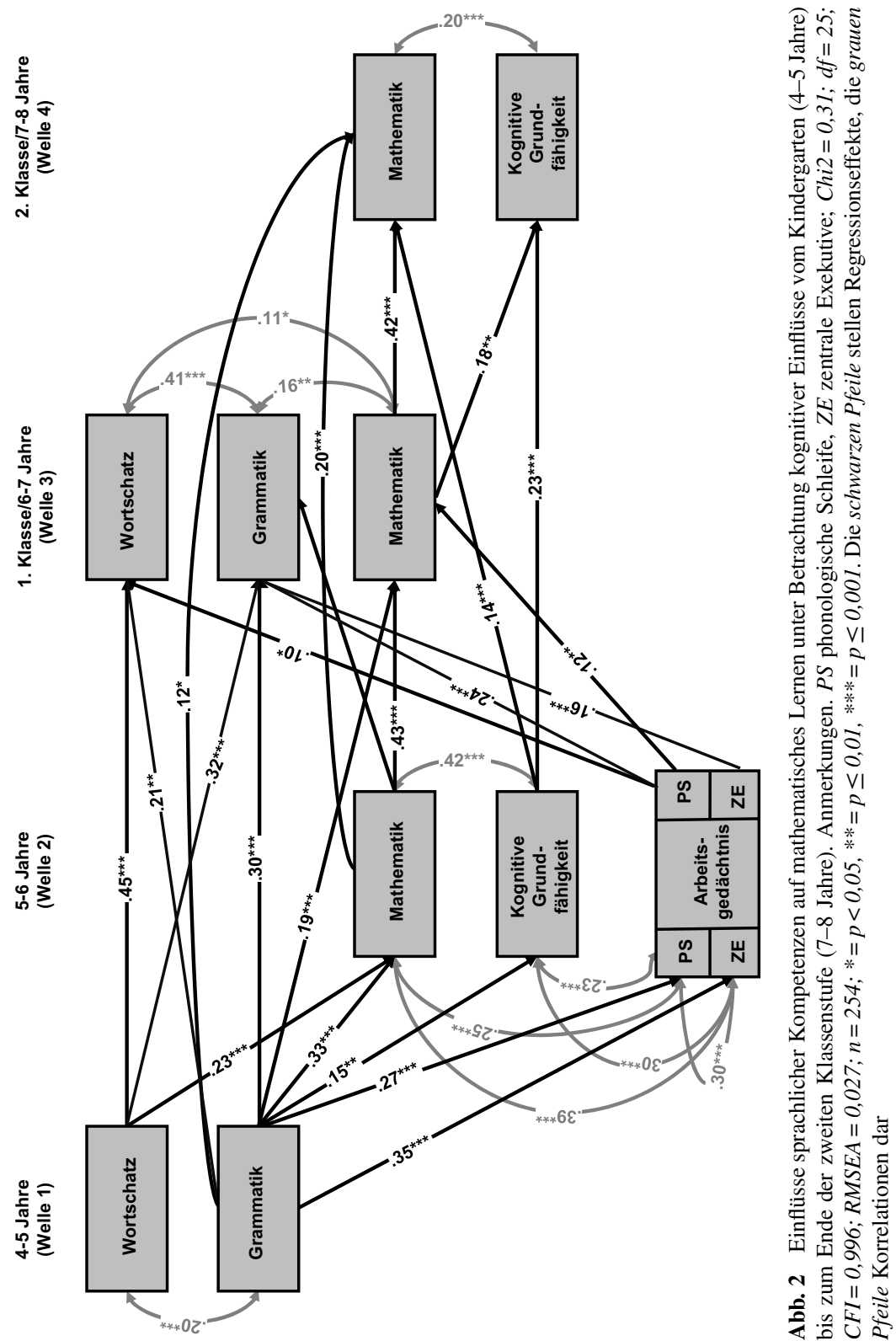
$S E(B)=0,03, p<0,001,95 \%$, KI für B $[0,16,0,30])$ sowie zur Kontrolle hinzugezogene weitere sprachliche Kompetenzen (Textverständnis Welle 4, 7/8 Jahre: $B=0,06$, $S E(B)=0,02, p<0,001,95 \%$, KI für B $[0,04,0,10])$ als Mediatoren. Kognitive Fähigkeiten besitzen jedoch keinerlei Einfluss. Vertiefende Analysen zeigen, dass auch der Wortschatz der ersten Klassenstufe nur indirekt die Mathematikleistungen der zweiten Klassenstufe beeinflusst. Auch hier wirken als Mediatoren einzig mathematische Kompetenzen der ersten Klassenstufe $(B=0,20, S E(B)=0,04, p<0,001,95 \%$, KI für B $[0,13,0,28])$ sowie das Textverständnis $(B=0,05, S E(B)=0,01, p<0,001$, $95 \%$, KI für B $[0,02,0,08])$ als sprachliches Kontrollmaß.

\subsection{Beziehungen zwischen sprachlichen Kompetenzen und kognitiven Fähigkeiten}

Vorschulisch gemessene Leistungen des Grammatikverständnisses (4-5 Jahre) beeinflussen positiv phonologische $(0,27 * * *)$ wie auch zentral-exekutive Arbeitsgedächtnisleistungen $(0,35 * * *)$ kurz vor Schuleintritt (5-6 Jahre). Diese wiederum wirken sich prädiktiv (phonologische Schleife: 0,24***; zentrale Exekutive: 0,16***) auf das Grammatikverständnis des ersten Schuljahres aus. Der Einfluss des frühen Grammatikverständnisses (4-5 Jahre) auf kognitive Grundfähigkeiten des Vorschulalters (5-6 Jahre) kann unter Kontrolle durchgeführter Mediationsanalysen einzig indirekt über die Zusammenhänge mit dem zur Kontrolle eingesetztem Sprachmaß der phonologischen Bewusstheit $(B=0,11, S E(B)=0,03, p<0,001,95 \%$, KI für B $[0,06,0,17])$ sowie der zentralen Exekutive $(B=0,08, S E(B)=0,02, p<0,001$, $95 \%$, KI für B $[0,04,0,13])$ erklärt werden. Auch zeigen sich keinerlei direkte Zusammenhänge zwischen dem Arbeitsgedächtnis bzw. den kognitiven Grundfähigkeiten und der Entwicklung des rezeptiven Wortschatzes im Vorschulalter und in der Grundschule. Diese werden lediglich über andere sprachliche sowie kognitive Maße mediiert.

\subsection{Beziehungen zwischen mathematischen Kompetenzen und kognitiven Fähigkeiten}

Innerhalb des Altersbereichs zwischen 5 und 8 Jahren zeigen sich wechselseitige Einflüsse zwischen den einzelnen Messungen mathematischer Kompetenzen und den kognitiven Grundfähigkeiten: Neben den Zusammenhängen beider Kompetenzbereiche innerhalb einzelner Altersstufen indizieren diese einen Einfluss vorschulisch gemessener kognitiver Grundfähigkeiten auf die mathematischen Leistungen der zweiten Klassenstufe $(0,14 * * *)$. Ebenso scheinen mathematische Leistungen des ersten Schuljahres die kognitiven Grundfähigkeiten der zweiten Klassenstufe vorherzusagen $(0,18 * *)$. Daneben zeigt sich ein Einfluss der vorschulisch gemessenen phonologischen Schleife auf mathematische Leistungen der ersten Klassenstufe $\left(0,12^{* *}\right)$. Die zentrale Exekutive dagegen scheint auf diese keinen direkten Einfluss zu nehmen. 


\subsection{Die Rolle der Sprache im Zusammenspiel mathematischer und kognitiver Fähigkeiten}

In einem weiteren Schritt werden die angenommenen wechselseitigen Entwicklungszusammenhänge zwischen mathematischen Kompetenzen und der Leistungsfähigkeit direkter (phonologische Schleife, zentrale Exekutive) bzw. indirekter (kognitive Grundfähigkeiten) Maße des Arbeitsgedächtnisses (vgl. 4.4) auf indirekte Effekte überprüft. Im Rahmen der Überprüfung wird speziell die Rolle der sprachlichen Kompetenzen innerhalb der angenommenen Entwicklungszusammenhänge zwischen mathematischen Kompetenzen und der Leistungsfähigkeit des Arbeitsgedächtnisses geklärt. Dazu wurden bestehende längsschnittliche Effekte durch Berechnung vertiefender Mediationsanalysen derselben Daten kontrolliert (vgl. Abb. 3, 4, 5, 6 und 7). Querschnittliche Mediationsanalysen der zweiten und vierten Erhebungswelle konnten aufgrund paralleler Messungen mathematischer Kompetenzen und kognitiver Fähigkeiten hinzugenommen werden (vgl. Abb. 3 und 7).

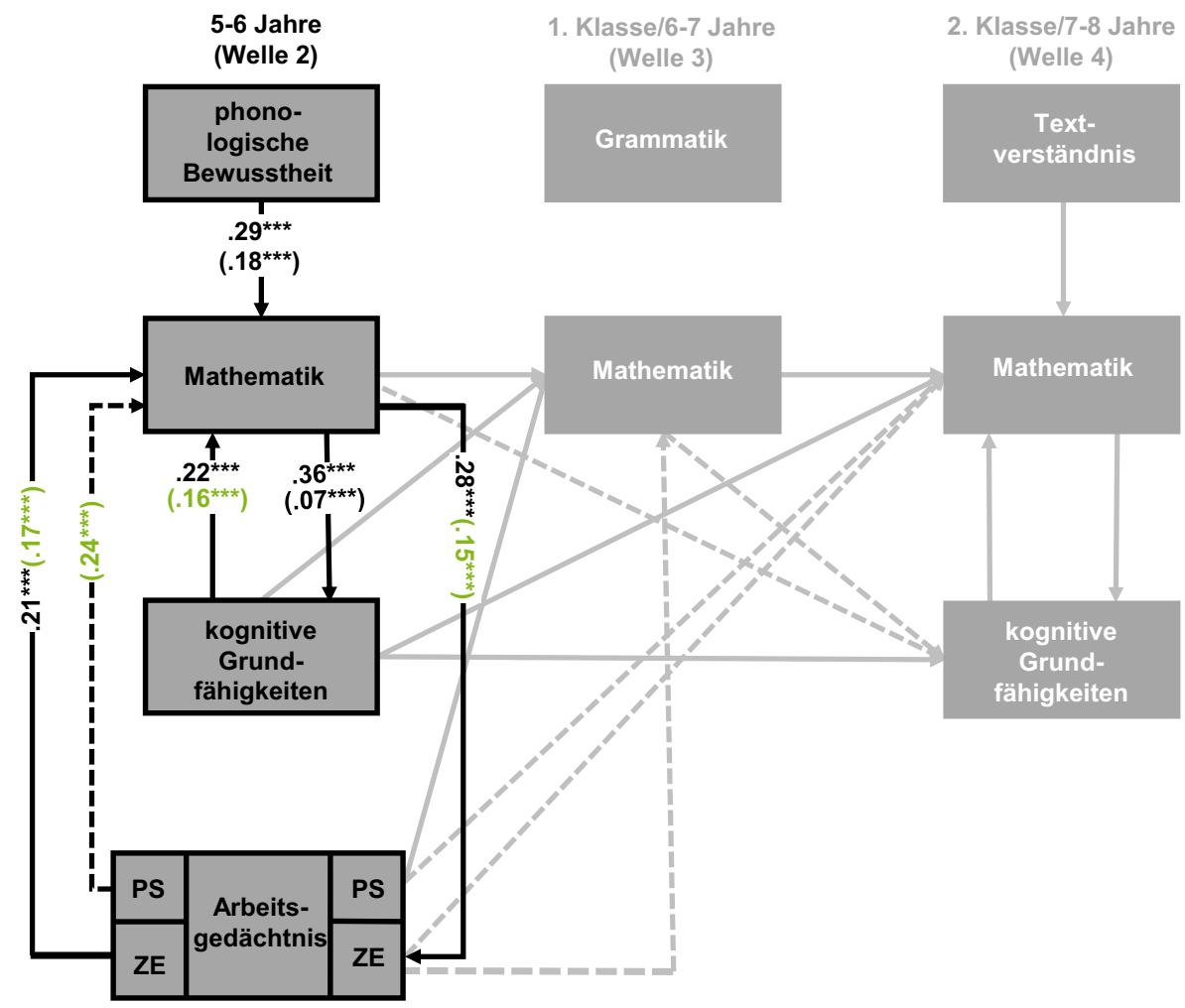

Abb. 3 Direkte und indirekte Effekte innerhalb der Welle 2 (5-6 Jahre) im Zusammenspiel mathematischer Kompetenzen und kognitiver Fähigkeiten unter Beachtung sprachlicher Mediatoren. Anmerkungen. Überprüfung der im Gesamtmodell (vgl. Abb. 2) bestehenden Pfade anhand querschnittlicher Mediationsanalysen mit Mplus. PS phonologische Schleife, ZE zentrale Exekutive. Gestrichelte Linien bilden indirekte, durchgezogene Linien direkte Effekte ab. In Klammern gesetzte Werte stellen indirekte Einflüsse dar. Hervorgehobene Werte in Klammern verdeutlichen indirekte Einflüsse, welche (z. T.) durch sprachliche Kompetenzen mediiert werden (vgl. Tab. 2, Welle 2) 
Die Richtung von Zusammenhängen wurde im Theorieteil (vgl. 1.5) begründet, wodurch sich Einzelmodelle beider möglichen Richtungen (mathematische Kompetenzen auf kognitive Fähigkeiten vs. kognitive Fähigkeiten auf mathematische Kompetenzen) ableiten lassen. Direkte und indirekte Zusammenhänge wurden unter Kontrolle weiterer sprachlicher Kompetenzen dieser beiden Erhebungswellen (Welle 2: phonologische Bewusstheit; Welle 4: Textverständnis) identifiziert.

Innerhalb beider Erhebungswellen der querschnittlichen Analysen (Welle 2, Welle 4) lassen sich direkte wechselseitige Zusammenhänge zwischen kognitiven Fähigkeiten und mathematischen Kompetenzen identifizieren (vgl. Abb. 3 und 7). So beeinflussen sowohl vorhandene kognitive Grundfähigkeiten innerhalb beider Erhebungswellen direkt mathematische Kompetenzen (Welle 2: 0,22***; Welle 4: $\left.0,25^{* * *}\right)$ wie auch mathematische Kompetenzen direkt die Ausprägung kognitiver Grundfähigkeiten (Welle 2: 0,36***; Welle 4: 0,32***). Vorhandene Wech-

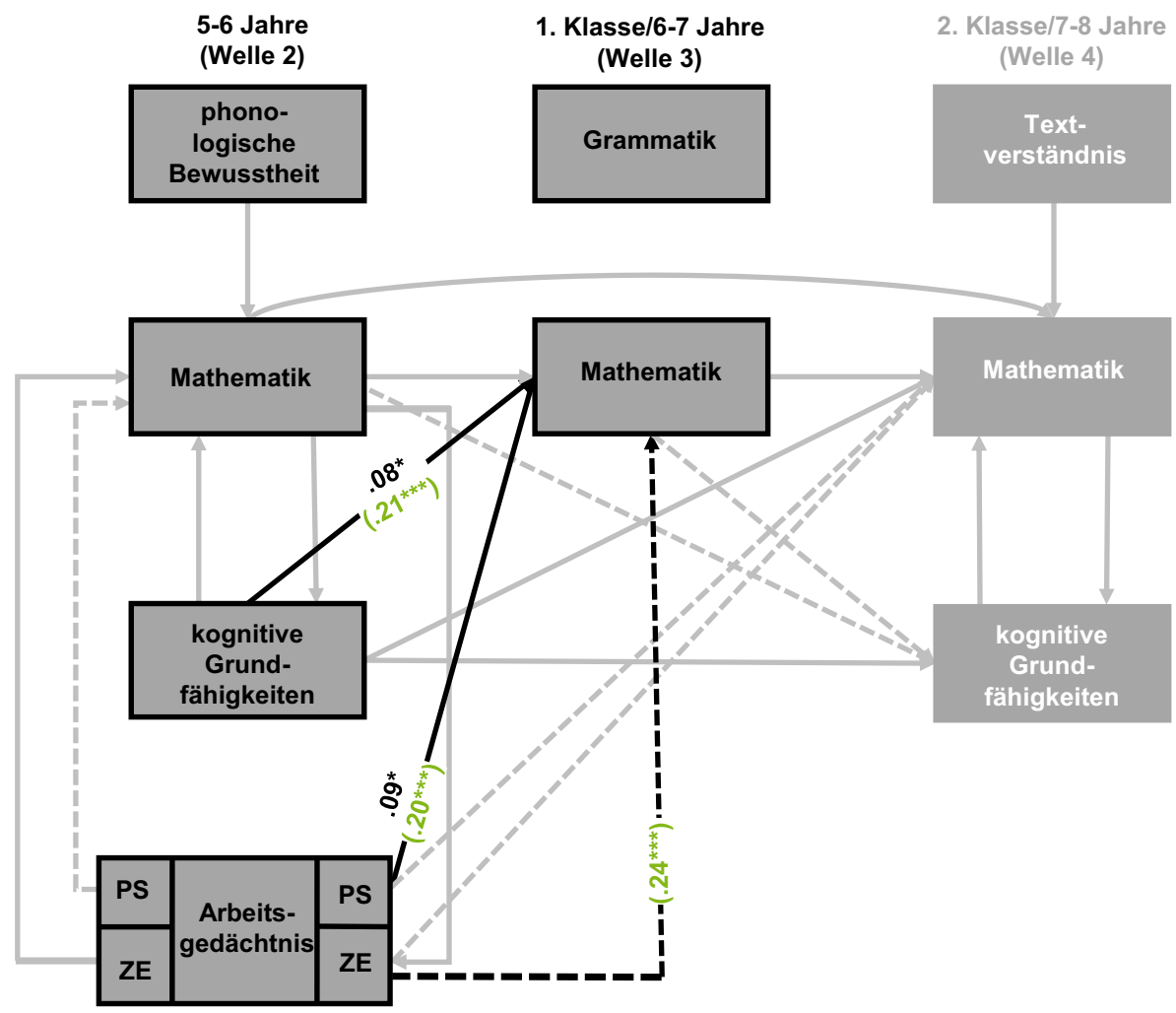

Abb. 4 Direkte und indirekte Effekte zwischen Welle 2 (5-6 Jahren) und Welle 3 (6-7 Jahren) im Zusammenspiel mathematischer Kompetenzen und kognitiver Fähigkeiten unter Beachtung sprachlicher Mediatoren. Anmerkungen. Überprüfung der im Gesamtmodell (vgl. Abb. 2) bestehenden Pfade anhand längsschnittlicher Mediationsanalysen mit Mplus. PS phonologische Schleife, ZE zentrale Exekutive. Gestrichelte Linien bilden indirekte, durchgezogene Linien direkte Effekte ab. In Klammern gesetzte Werte stellen indirekte Einflüsse dar. Hervorgehobene Werte in Klammern verdeutlichen indirekte Einflüsse, welche (z. T.) durch sprachliche Kompetenzen mediiert werden (vgl. Tab. 2, Welle 2). Auf die Abbildung des Wortschatzes im vorliegenden Modell wurde aufgrund fehlender Bedeutung als Mediator im Zusammenspiel zwischen mathematischen und kognitiven Kompetenzen verzichtet 
selbeziehungen bilden innerhalb der zweiten Erhebungswelle (vgl. Abb. 3) auch direkte Zusammenhänge zwischen Leistungen der zentralen Exekutive und mathematischen Kompetenzen ab $(0,21 * * * / 0,28 * * *)$. Einzig die phonologische Schleife im Vorschulalter hängt nur indirekt über sprachliche Kompetenzen und kognitive Fähigkeiten (kognitive Grundfähigkeiten, zentrale Exekutive) mit mathematischen Leistungen der gleichen Altersstufe zusammen. Innerhalb beider Erhebungswellen stellen sich auch sprachliche Maße als Mediatoren zwischen kognitiven Fähigkeiten und mathematischen Kompetenzen dar. Vorhandene Mediationseffekte werden aufgrund der Komplexität in Tab. 2 gesondert dargestellt, wobei sprachliche Maße hervorgehoben werden. So spielt innerhalb der zweiten Erhebungswelle die phonologische Bewusstheit, innerhalb der vierten Erhebungswelle das Textverständnis eine vermittelnde Rolle.

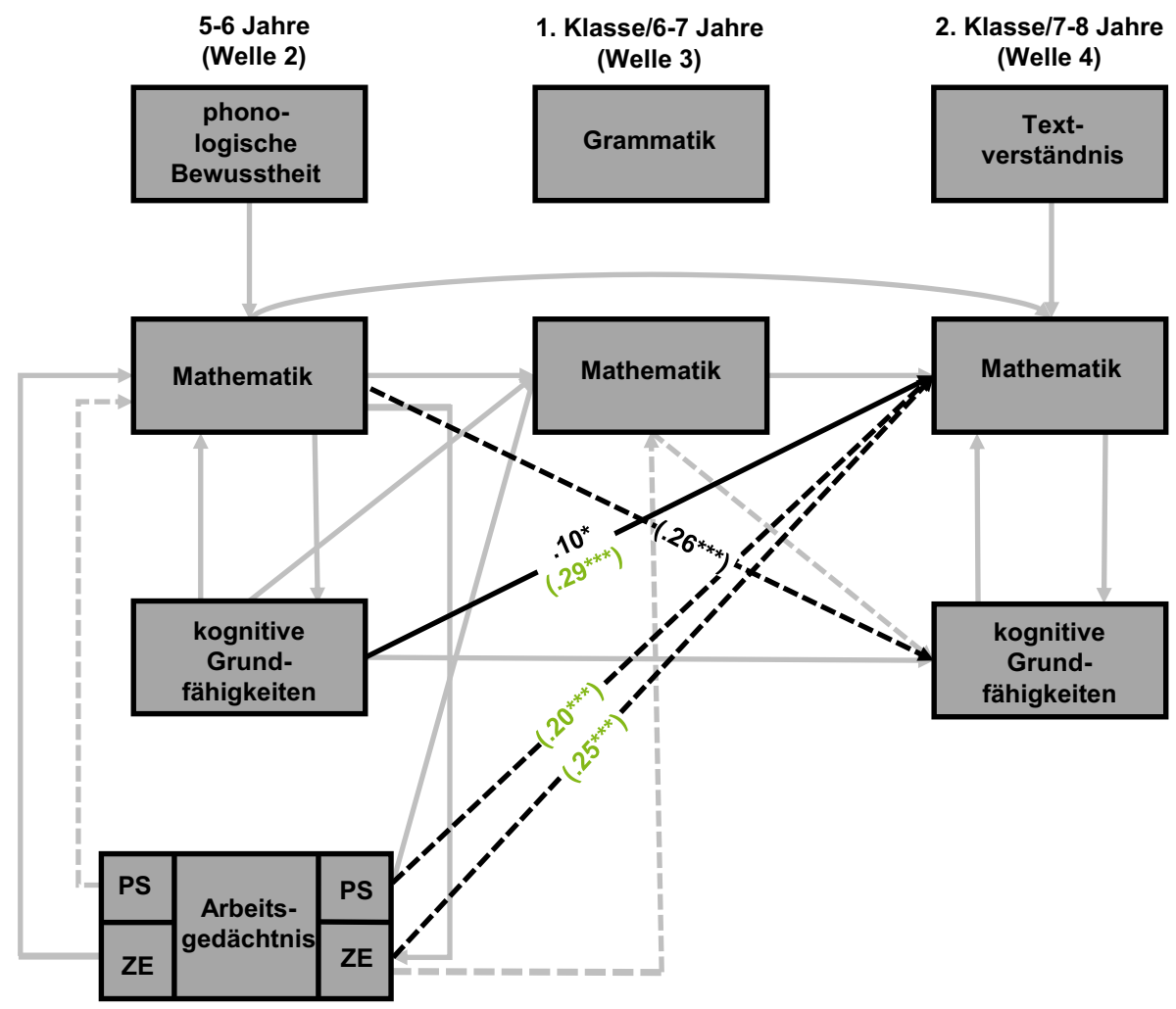

Abb. 5 Direkte und indirekte Effekte zwischen Welle 2 (5-6 Jahren) und Welle 4 (7-8 Jahren) im Zusammenspiel mathematischer Kompetenzen und kognitiver Fähigkeiten unter Beachtung sprachlicher Mediatoren. Anmerkungen. Überprüfung der im Gesamtmodell (vgl. Abb. 2) bestehenden Pfade anhand längsschnittlicher Mediationsanalysen mit Mplus. PS phonologische Schleife, ZE zentrale Exekutive. Gestrichelte Linien bilden indirekte, durchgezogene Linien direkte Effekte ab. In Klammern gesetzte Werte stellen indirekte Einflüsse dar. Hervorgehobene Werte in Klammern verdeutlichen indirekte Einflüsse, welche (z. T.) durch sprachliche Kompetenzen mediiert werden (vgl. Tab. 2, Welle 2). Auf die Abbildung des Wortschatzes im vorliegenden Modell wurde aufgrund fehlender Bedeutung als Mediator im Zusammenspiel zwischen mathematischen und kognitiven Kompetenzen verzichtet 
Längsschnittlich bestätigt sich, dass vorschulische kognitive Grundfähigkeiten die mathematischen Leistungen der ersten (vgl. Abb. 4) und zweiten Klassenstufe (vgl. Abb. 5) direkt beeinflussen. Dagegen besteht der Einfluss vorschulisch gemessener mathematischer Kompetenzen auf kognitive Grundfähigkeiten im zweiten Schuljahr nur indirekt. Als Mediatoren fungieren die vorschulischen kognitiven Grundfähigkeiten und mathematische Kompetenzen in der ersten und zweiten Klassenstufe. Ähnliches zeigt sich für den Einfluss mathematischer Kompetenzen im ersten Schuljahr auf die kognitiven Grundfähigkeiten der zweiten Klassenstufe (vgl. Abb. 6). Dieser Effekt lässt sich einzig über die mathematischen Kompetenzen der zweiten Klassenstufe erklären (vgl. Tab. 2). Vereinzelt stellen sich auch die phonologische Bewusstheit und/oder die Grammatik als Mediatoren zwischen kognitiven Fähigkeiten (kognitive Grundfähigkeiten, phonologische Schleife, zentrale Exekutive) und mathematischen Kompetenzen der ersten und zweiten Klassenstufe dar.

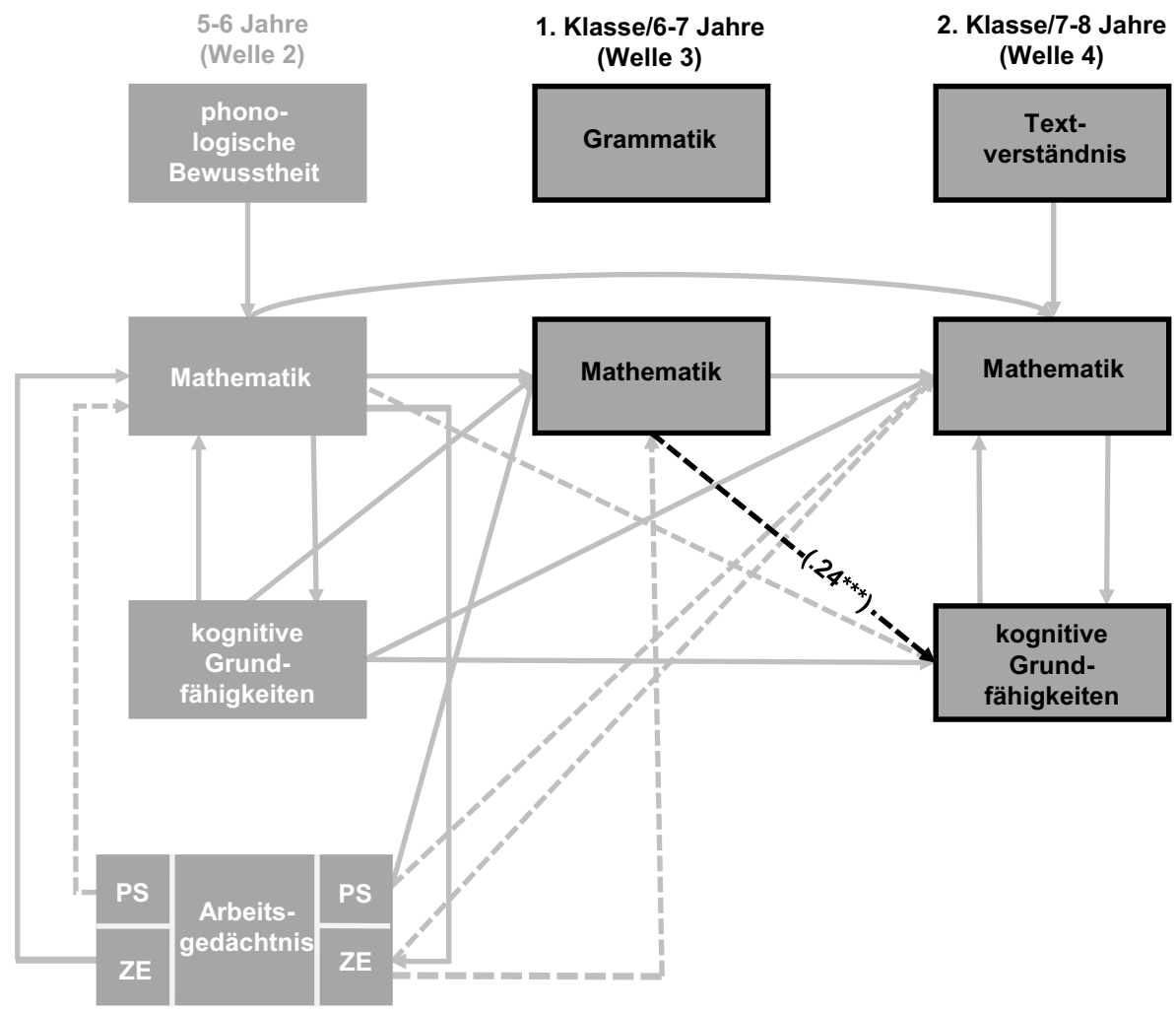

Abb. 6 Direkte und indirekte Effekte zwischen Welle 3 (6-7 Jahren) und Welle 4 (7-8 Jahren) im Zusammenspiel mathematischer Kompetenzen und kognitiver Fähigkeiten unter Beachtung sprachlicher Mediatoren. Anmerkungen. Überprüfung der im Gesamtmodell (vgl. Abb. 2) bestehenden Pfade anhand längsschnittlicher Mediationsanalysen mit Mplus. PS phonologische Schleife, ZE zentrale Exekutive. Gestrichelte Linien bilden indirekte, durchgezogene Linien direkte Effekte ab. In Klammern gesetzte Werte stellen indirekte Einflüsse dar. Hervorgehobene Werte in Klammern verdeutlichen indirekte Einflüsse, welche (z. T.) durch sprachliche Kompetenzen mediiert werden (vgl. Tab. 2, Welle 2). Auf die Abbildung des Wortschatzes im vorliegenden Modell wurde aufgrund fehlender Bedeutung als Mediator im Zusammenspiel zwischen mathematischen und kognitiven Kompetenzen verzichtet 


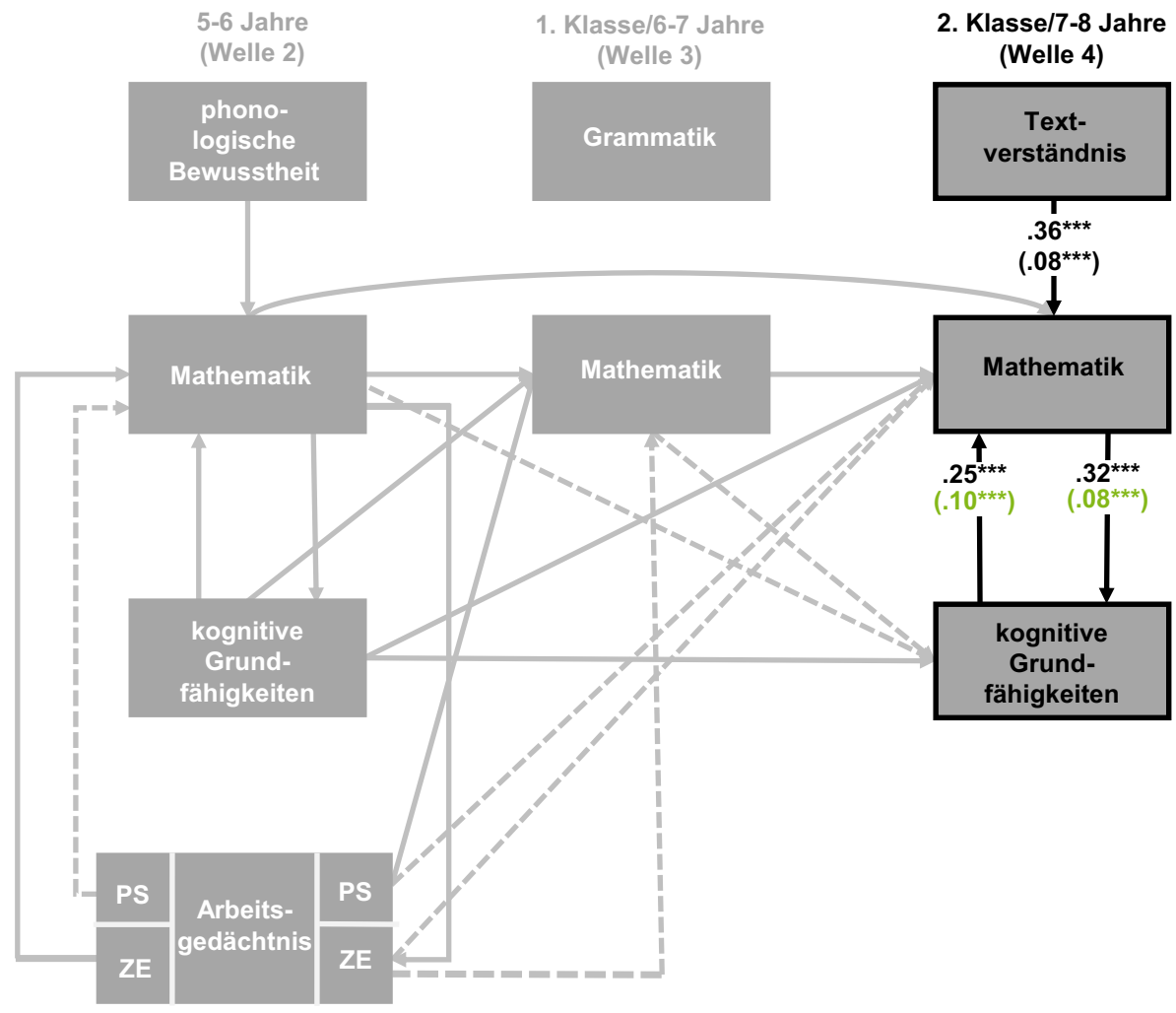

Abb. 7 Direkte und indirekte Effekte innerhalb der Welle 4 (7-8 Jahre) im Zusammenspiel mathematischer Kompetenzen und kognitiver Fähigkeiten unter Beachtung sprachlicher Mediatoren. Anmerkungen. Überprüfung der im Gesamtmodell (vgl. Abb. 2) bestehenden Pfade anhand querschnittlicher Mediationsanalysen mit Mplus. PS phonologische Schleife, ZE zentrale Exekutive. In Klammern gesetzte Werte stellen indirekte Einflüsse dar. Hervorgehobene Werte in Klammern verdeutlichen indirekte Einflüsse, welche (z. T.) durch sprachliche Kompetenzen mediiert werden (vgl. Tab. 2, Welle 2)

\section{Diskussion}

Ziel der vorliegenden Untersuchung war es, vermittelnde Effekte sprachlicher Einflüsse auf mathematisches Lernen des Entwicklungszeitraums vom Vorschulalter bis in die Grundschule zu identifizieren. Dabei galt es, neben der Aufdeckung direkter Effekte sprachlicher Kompetenzen auf mathematisches Lernen auch die ihnen zugrundeliegenden Interdependenzen aufzudecken. Durch Nutzung vorhandener Kompetenzdaten des Nationalen Bildungspanels war es möglich, zusätzlich die Rolle des kognitiven Systems des Arbeitsgedächtnisses innerhalb des Zusammenspiels sprachlicher und mathematischer Entwicklung näher zu betrachten. Dabei sollte insbesondere auch die Rolle sprachlicher Kompetenzen innerhalb mit mathematischem Lernen in Verbindung stehenden kognitiven Vorgängen des Arbeitsgedächtnisses offengelegt werden. Zudem wurden weitere, die Sprache beeinflussende, Indikatoren 


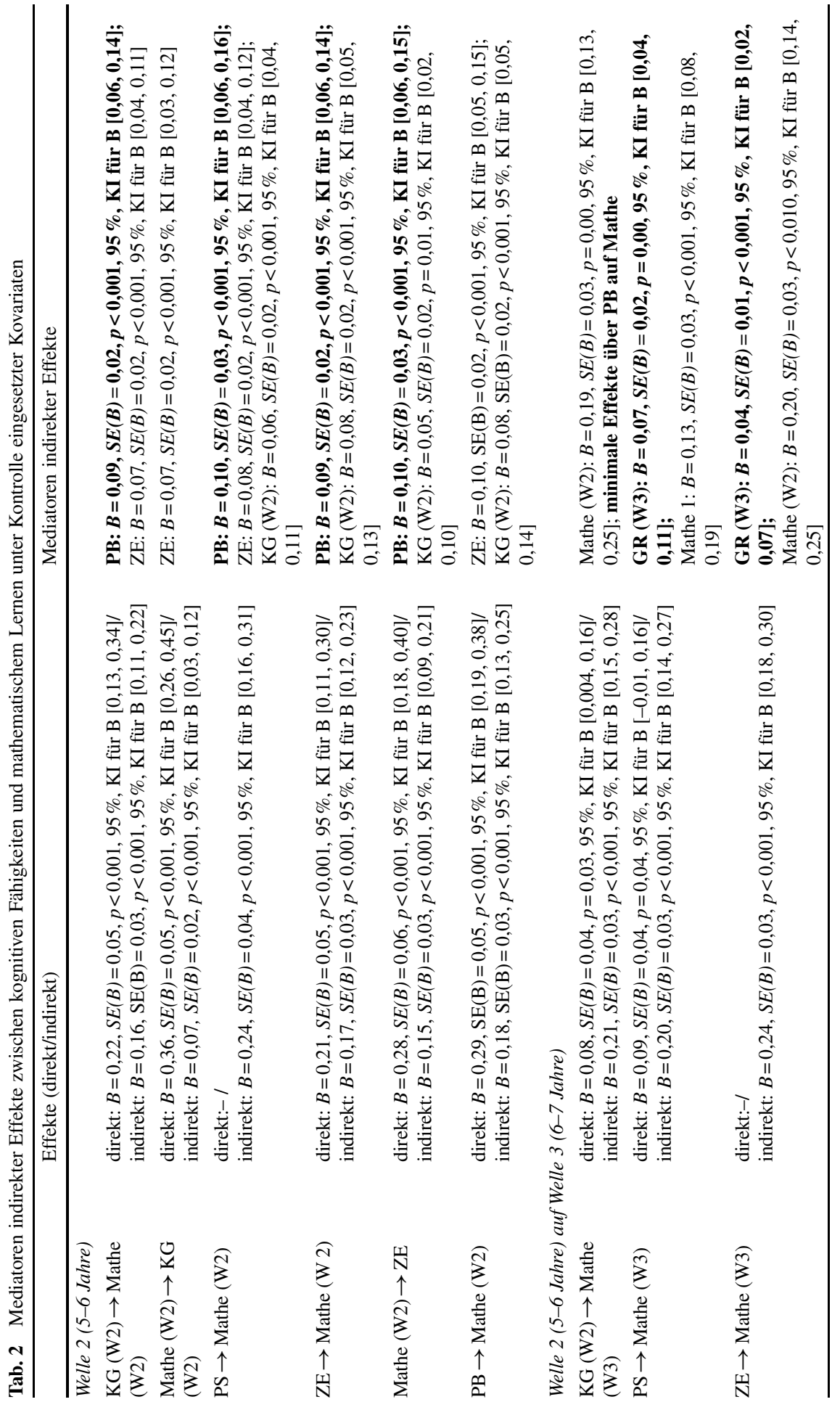




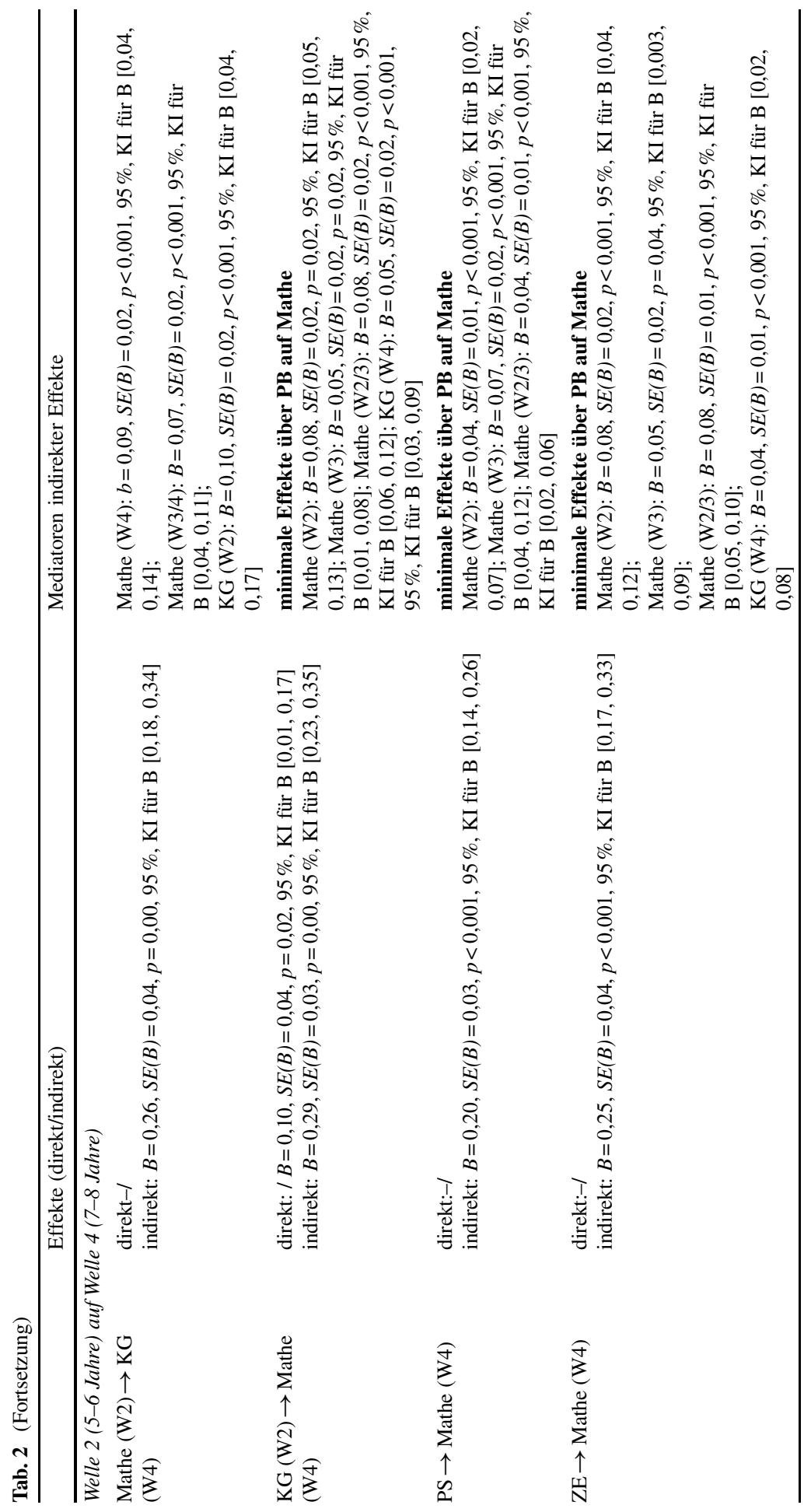




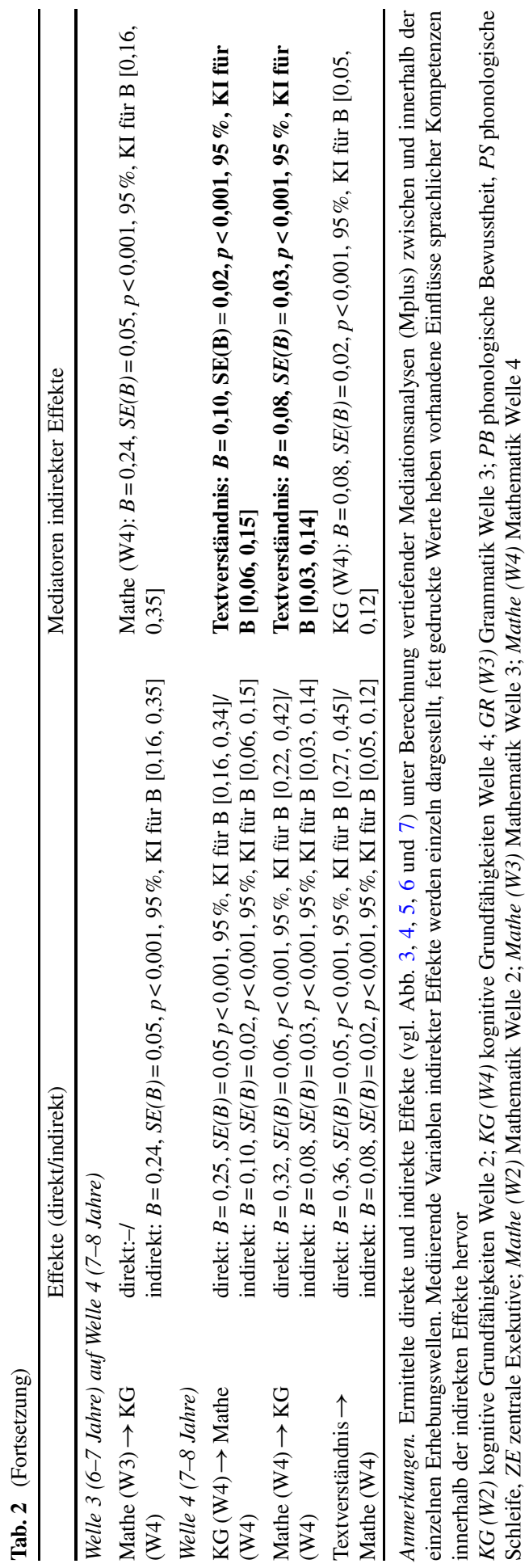


(sprachliche Lernschwäche, die zuhause gesprochene Sprache, Migrationshintergrund, sozioökonomischer Hintergrund) kontrolliert.

Die Ergebnisse legen nahe, dass sprachliche Kompetenzen und dabei insbesondere das Grammatikverständnis, das mathematische Lernen langfristig beeinflussen. Der Wortschatz scheint dagegen nur beim frühen mathematischen Kompetenzerwerb relevant zu sein. Zwar hängt dieser innerhalb der einzelnen Altersstufen im Grundschulalter mit der Ausprägung mathematischer Leistungen zusammen, nimmt aber - im Gegensatz zu den grammatikalischen Kompetenzen - keinen direkten Einfluss auf die weitere mathematische Entwicklung im Schulalter. Dieser Befund deutet auf eine höhere sprachliche Komplexität beim mathematischen Lernen im Schul- gegenüber dem Vorschulalter hin, wenn mathematische Fachwörter sowie die Unterscheidung von Alltags- und Bildungssprache an Bedeutung gewinnen (u. a. Bochnik 2017; Prediger et al. 2019).

Zudem scheint das Grammatikverständnis im Vorschulalter eng im Zusammenhang mit der Leistungsfähigkeit des Arbeitsgedächtnisses zu stehen. Ein gutes Arbeitsgedächtnis begünstigt einerseits die Entwicklung weiterer mit dem mathematischen Lernen vorteilhaft in Zusammenhang stehender grammatikalischer Sprachkompetenzen im Grundschulalter. Zudem scheinen bereits bestehende vorschulische Kompetenzen in der Grammatik die Leistungsfähigkeit dieses Systems durch eine Entlastung dessen begrenzter Kapazität zu verbessern (vgl. Hasselhorn und Gold 2006). Nach unserem Wissen konnte dies in vorherigen Untersuchungen bisher nur für den Wortschatz nachgewiesen werden (Gathercole et al. 1992). Auch bestätigt sich der Einfluss des vorschulischen Arbeitsgedächtnisses auf die Entwicklung mathematischer Kompetenzen im Vor- und Grundschulalter (vgl. Grube und Seitz-Stein 2012).

Die Ergebnisse vertiefender Mediationsanalysen heben weitere Funktionen von Sprache hervor, welche in die kognitiven Prozesse mathematischen Lernens eingebunden scheinen. Aufgedeckte Mediationsprozesse im Aufbau mathematischen Lernens (d.h. Sprache als Mediator zwischen der kognitiven Leistungsfähigkeit des Arbeitsgedächtnisses und den mathematischen Kompetenzen aber auch die kognitive Leistungsfähigkeit des Arbeitsgedächtnisses als Mediator für den Einfluss von Sprache auf mathematische Kompetenzen) weisen auf unterschiedliche Prozesse innerhalb des komplexen Zusammenspiels aller drei Domänen - Sprache, Mathematik und Arbeitsgedächtnis - hin. Auch hier bestätigt sich insbesondere die Relevanz des Grammatikverständnisses: Erstens spielen sprachliche Kompetenzen für die vorschulische Ausbildung von Gedächtnisfunktionen, wie sie für die Zahlenverarbeitung notwendig sind (vgl. von Aster 2013) eine Rolle. Dabei zeigt sich überraschenderweise nur ein Einfluss des Grammatikverständnisses. Der allgemeine Wortschatz hingegen erscheint vergleichsweise irrelevant. Hier zeigt sich die fachliche Spezifität des Wortschatzes, welcher bereits für die Entwicklung des Zahlenverständnisses, insbesondere der Zahlwortreihe, erforderlich ist (Schindler et al. 2019). Zweitens kann der Einfluss der Sprache innerhalb der Wechselbeziehung zwischen Arbeitsgedächtnis und mathematischen Kompetenzen (vgl. Tab. 2) auch auf eine mögliche vorschulische Nutzung sprachlicher Kompetenzen zur (Zwischen-)Speicherung mathematischer Inhalte hinweisen (Lorenz 2012). Im weiteren Grundschulbereich sind diese Mediationseffekte durch Sprache rückläufig. Die Rückläufigkeit sprachlicher 
Mediationseffekte innerhalb dieser Altersstufe legt nahe, dass die Möglichkeit, mathematische Inhalte sprachlich zu speichern, mit ansteigendem Alter seltener von den Kindern genutzt wird. Diese Beobachtung wäre durch eine zunehmende Automatisierung innerhalb mathematischer Lernprozesse (ebd.) zu erklären. Drittens scheinen die bestehenden sprachlichen Fähigkeiten im Vorschulalter eine allgemeine Voraussetzung darzustellen, mathematische Aufgabenstellungen im Vor- und Grundschulalter besser zu verarbeiten (s. oben). Darauf weisen sowohl der direkte Einfluss von Sprache auf mathematische Kompetenzen wie aber auch die mediierten Effekte der Grammatikleistungen auf mathematische Kompetenzen über kognitive Prozesse des Arbeitsgedächtnisses hin. Die Größe des Wortschatzes scheint auch hier, im Gegensatz zur Grammatik, nicht relevant. Die starke Gewichtung des Einflusses grammatikalischer Fähigkeiten auf mathematische Lernprozesse steht in Übereinstimmung mit vorherigen Befunden zum Lernen im Mathematikunterricht, welche speziell Kompetenzen komplexerer sprachlicher Register wie Fach- oder Bildungssprache als Voraussetzung für erfolgreiche mathematische Leistungen im Grundschulalter identifizieren konnten (z. B. Paetsch 2016; Prediger et al. 2019).

Zudem erlangen kognitive Fähigkeiten auch unabhängig von den sprachlichen Kompetenzen einen großen Einfluss zur Erklärung mathematischen Lernens im Vorund Grundschulalter. Dies belegen die Ergebnisse unabhängig davon, ob es sich um direkte Maße des Arbeitsgedächtnisses handelt oder indirekte, welche auch als kognitive Fähigkeiten im weiteren Sinne interpretiert werden können. Erstere zeigen im Einklang mit vorherigen Untersuchungen (z.B. Ehlert 2007) altersabhängige Veränderungen, welche auf Automatisierungen innerhalb mathematischer Lernprozesse schließen lassen (ebd.). Eine Verbesserung der Leistungsfähigkeit kognitiver Prozesse durch eine Kapazitätsentlastung im Schulalter lässt sich jedoch innerhalb dieser Studie nicht direkt durch zuvor bestehendes mathematisches Wissen erklären. Dies konnte eigentlich aufgrund von Ergebnissen einer Studie zu kreuzverzögerten Effekten zwischen mathematischen und kognitiven Fähigkeiten im Schulalter erwartet werden (Hoese 2017). Vertiefende Analysen zeigen jedoch, dass Einflüsse vorschulischer Mathematikleistungen auf kognitive Grundfähigkeiten in der zweiten Klassenstufe über den Zusammenhang mit vorschulischen kognitiven Grundfähigkeiten wie auch über späteres mathematisches Wissen mediiert werden. Sprachliche Kompetenzen spielen - wie bereits erwähnt - als Mediatoren innerhalb dieses längsschnittlichen Beziehungsmusters kaum eine Rolle, sondern nur für den starken Zusammenhang, welcher zwischen kognitiven Fähigkeiten und mathematischen Kompetenzen innerhalb der einzelnen Altersstufe direkt vorhanden ist. Insgesamt stellt sich zudem heraus, dass frühe mathematische Kompetenzen, unabhängig von den gesamten beschriebenen Interdependenzen, ihren direkten Einfluss auf mathematisches Lernen bis ins weitere Grundschulalter beibehalten (vgl. auch Krajewski 2014). Die adäquate Entwicklung dieser frühen mathematischen Kompetenzen wird jedoch von zahlreichen sprachlichen und kognitiven Fähigkeiten mitbeeinflusst. 


\section{Limitationen}

Die Abbildung komplexer Beziehungsmuster umfassender Kompetenzen im Vorschul- und Schulalter, unter Verwendung eines zur Verfügung stehenden Datensatzes, erforderte einige Kompromissbereitschaften: Einerseits berücksichtigen die Messungen von Sprache, Mathematik und Arbeitsgedächtnis der Startkohorte 2 des NEPS keine kontinuierliche Erhebung aller hier interessierenden Parameter zu jedem Zeitpunkt, was Aussagen bidirektionaler Zusammenhänge der drei Kompetenzen über alle Altersstufen hinweg ausschloss. Speziell das Arbeitsgedächtnis wurde direkt nur im Rahmen einer altersspezifischen Erhebung zur sprachlichen Informationsverarbeitung kurz vor Schuleintritt erfasst, was auch die fehlende Messung der visuellräumlichen Komponente erklärt. Zudem wiesen die direkten Maße der zentralen Exekutive aufgrund des Schwierigkeitsgrades des Tests eine eingeschränkte interne Konsistenz auf. Genauere und wiederholte Maße der zentralen Exekutive (inklusive einer visuell-räumlichen Speicherkomponente) konnten dadurch nur auf Grundlage indirekter Messungen über kognitive Grundfähigkeiten gewonnen werden. Deren vollständige Abgrenzung vom ursprünglichen Messkonstrukt kann aufgrund möglicher Konfundierungen jedoch nicht garantiert werden. Aufgrund der Abbildung mathematischer Kompetenzen als eindimensionales Konstrukt konnten keine Aussagen über einzelne mathematische Teilbereiche getroffen werden, wie dies für Sprache möglich war. Durch den Einbezug der Konzeption des Mathematical Literacy in das Messkonstrukt mathematischer Kompetenz bei NEPS (vgl. 3.2) ist zudem automatisch eine mehr oder weniger ausgeprägte Sprachnähe gegeben.

\section{Fazit}

Die Ergebnisse der vorliegenden Untersuchung lassen ein komplexes Zusammenspiel deutlich werden, welches sich hinter dem Einfluss sprachlicher Kompetenzen auf mathematisches Lernen abzeichnet. Dabei wird der Einfluss von Sprache in unterschiedlichen Funktionen deutlich, welche sich - neben ihrem direkten Einfluss auf mathematisches Lernen - sowohl in der ,,Voraussetzung“ wie auch in der „Mediation" kognitiver, mit mathematischem Lernen in Zusammenhang stehender Prozesse, erkennen lässt. Auch wird die Bedeutung früher mathematischer Kompetenzen selbst für späteres mathematisches Lernen durch ihre engen Entwicklungszusammenhänge mit sprachlichen Kompetenzen direkt untermauert. Dabei scheinen bereits komplexere sprachliche Fertigkeiten im Vorschulalter dominant. Die Rolle der Sprache für frühe mathematische Kompetenzen wird zudem durch deren spezielle Bedeutung innerhalb kognitiver Prozesse des Erwerbs früher mathematischer Kompetenzen verstärkt. Aufgrund ihres Einflusses über die zahlreichen beschriebenen Ebenen innerhalb mathematischer Lernprozesse scheint Sprache somit - auch unter Kontrolle weiterer, die Sprachkompetenz beeinflussender Faktoren - einen wesentlichen Bestandteil mathematischen Lernens auszumachen. Dies legt die Notwendigkeit nahe, die unterschiedlichen Funktionen von Sprache innerhalb des mathematischen Lernens bereits in vorschulischen und später in schulischen Lernprozessen zu berücksichtigen. Bereits bestehende erfolgreiche Ansätze im Vorschulalter durch eine 
integrierte Förderung von Sprache und Mathematik in Kita und Familie (z. B. Böning und Thöne 2017) sowie die Umsetzung eines entsprechend sprachsensiblen Unterrichts in Schulen (z. B. Sprachsensible Schulentwicklung 2017) bestätigen die Forderung einer Etablierung und Weiterentwicklung dieses Bereichs. Dabei sollte gerade im Hinblick auf die notwendige Anschlussfähigkeit an schulisches Lernen (z. B. Gasteiger 2017) auch vorschulisch bereits einer Etablierung komplexerer sprachlicher Fähigkeiten in mathematische Lernsituationen Rechnung getragen werden. Insbesondere eine Fokussierung auf grammatikalische Strukturen scheint dabei bereits vorschulisch angeraten. Überdies würde die Beachtung einer ,Vielfalt von Sprache“ dem Einsatz eines breitgefächerten mathematischen Lernmaterials nur entsprechen (Tiedemann 2017). Bei der Auswahl dieses Materials sollten jedoch im Hinblick auf das kapazitätsbegrenzte und für mathematisches Lernen relevante Arbeitsgedächtnis Anpassungen vorgenommen werden, indem eine Überlastung der Ressourcen durch die Vermeidung unnötiger Informationen vermieden wird (Krajewski und Ennemoser 2010).

Funding Open Access funding provided by Projekt DEAL.

Open Access Dieser Artikel wird unter der Creative Commons Namensnennung 4.0 International Lizenz veröffentlicht, welche die Nutzung, Vervielfältigung, Bearbeitung, Verbreitung und Wiedergabe in jeglichem Medium und Format erlaubt, sofern Sie den/die ursprünglichen Autor(en) und die Quelle ordnungsgemäß nennen, einen Link zur Creative Commons Lizenz beifügen und angeben, ob Änderungen vorgenommen wurden.

Die in diesem Artikel enthaltenen Bilder und sonstiges Drittmaterial unterliegen ebenfalls der genannten Creative Commons Lizenz, sofern sich aus der Abbildungslegende nichts anderes ergibt. Sofern das betreffende Material nicht unter der genannten Creative Commons Lizenz steht und die betreffende Handlung nicht nach gesetzlichen Vorschriften erlaubt ist, ist für die oben aufgeführten Weiterverwendungen des Materials die Einwilligung des jeweiligen Rechteinhabers einzuholen.

Weitere Details zur Lizenz entnehmen Sie bitte der Lizenzinformation auf http://creativecommons.org/ licenses/by/4.0/deed.de.

\section{Literatur}

Abou-Koura, K., Perleth, C. (2005). Kognitiver Fähigkeitstest (KFT 1-2R): bislang unveröffentlichte Revision. Rostock: Universität Rostock.

de Abreu, E. P. M. J., Conway, A. R. A., \& Gathercole, S.E. (2010). Working memory and fluid intelligence in young children. Intelligence, 38(6), 552-561.

Adams, A.-M., \& Gathercole, S.E. (1996). Phonological working memory and spoken language development in young children. The Quarterly Journal of Experimental Psychology, 49A(1), 216-233.

von Aster, M. (2013). Wie kommen Zahlen in den Kopf und was kann sie daran hindern? Ein Modell der normalen und abweichenden Entwicklung zahlenverarbeitender Hirnfunktionen. In M. von Aster \& J.H. Lorenz (Hrsg.), Rechenstörungen bei Kindern: Neurowissenschaft, Psychologie, Pädagogik (S. 15-38). Göttingen: Vandenhoeck \& Ruprecht.

Baddeley, A. (2000). Working memory. Current Biology, 20(4), 136-140.

Berendes, K., Weinert, S., Zimmermann, S., \& Artelt, C. (2013). Assessing language indicators across the lifespan within the German National Educational Panel Study (NEPS). Journal for Educational Research Online, 5(2), 15-49.

Beyer, R., \& Gerlach, R. (2018). Sprache und Denken (2. Aufl.). Wiesbaden: Springer.

Bishop, D. V. (1989). TROG - Test for Reception of Grammar. Manchester, GB: Medical Research Council. 
Blossfeld, H.-P., Roßbach, H.-G., \& von Maurice, J. (2011). Education as a lifelong process. Zeitschrift Für Erziehungswissenschaften, 14, 19-34. Sonderheft.

Bochnik, K. (2017). Sprachbezogene Merkmale als Erklärung für Disparitäten mathematischer Leistung. Differenzierte Analysen im Rahmen einer Längsschnittstudie in der dritten Jahrgangsstufe. Münster: Waxmann.

Böning, D., \& Thöne, B. (2017). Integrierte Förderung von Sprache und Mathematik in Kita und Familie. In I. S. Schuler, C. Streit \& G. Wittmann (Hrsg.), Perspektiven mathematischer Bildung im Übergang vom Kindergarten zur Grundschule (S. 27-39). Wiesbaden: Springer.

Bortz, J., \& Döring, N. (2016). Forschungsmethoden und Evaluation: Für Human- und Sozialwissenschaft$\operatorname{ler}$ (5. Aufl.). Berlin: Springer.

Christ, O., \& Schlüter, E. (2012). Strukturgleichungsmodelle mit Mplus: Eine praktische Einführung. München: Oldenbourg.

Cornu, V., Schiltz, C., Martin, R., \& Hornung, C. (2017). Visuo-spatial abilities are key for young children's verbal number skills. Journal of Experimental Child Psychology, 166, 604-620. https://doi. org/10.1016/j.jecp.2017.09.006.

Diaz-Barriga Yanez, A., Carroll, D. J., \& Matthews, D. (2016). Exploring the role of working memory components in mathematical skills in 5-6 years old children. Sheffield: University of Sheffield.

Dunn, L.M., \& Dunn, D. M. (2007). Peabody picture vocabulary test, fourth edition (PPVT-4). Upper Saddle River: Pearson.

Ehlert, A. (2007). Arbeitsgedächtnis und Rechnen im Vorschulalter: Die Entwicklung eines Arbeitsgedächtnistests und eines Untersuchungsverfahrens für mathematische Kompetenzen zur Überprüfung des Einflusses des Arbeitsgedächtnisses nach Baddeley auf mathematische Fertigkeiten im Vorschulalter (Dissertation). Humboldt Universität, Berlin.

Fischer, L., Rohm, T., Gnambs, T., \& Carstensen, C.H. (2016). Linking the data of the competence test. Verfügbar unter. https://www.neps-data.de. Zugegriffen: 11. Dez. 2018.

Fox, A. V. (2006). TROG-D Test zur Überprüfung des Grammatikverständnisses. Idstein: Schulz-Kirchner.

Fricke, S., \& Schäfer, B. (2008). Test für phonologische Bewusstheitsfähigkeiten (TPB). Idstein: SchulzKirchner.

Ganzeboom, H.B.G. (2010). A new international socio-economic index [ISEI] of occupational status for the international standard classification of occupation 2008 [ISCO-08] constructed with data from the ISSP 2002-2007. Annual Conference of International Social Survey Programme, Lisbon.

Garson, G. D. (2015). Missing values analysis \& data imputation. North Carolina: Statistical Associates Publishing.

Gasteiger, H. (2017). Frühe mathematische Bildung - sachgerecht, kindgemäß, anschlussfähig. In S. Schuler, C. Streit \& G. Wittmann (Hrsg.), Perspektiven mathematischer Bildung im Übergang vom Kindergarten zur Grundschule (S. 9-26). Wiesbaden: Springer.

Gathercole, S. E., Willis, C. S., Emslie, H., \& Baddeley, A. D. (1992). Phonological memory and vocabulary development during the early school years: A longitudinal study. Developmental Psychology, 28(5), 887-898.

Götze, B., Hasselhorn, M., \& Kiese-Himmel, C. (2000). Phonologisches Arbeitsgedächtnis, Wortschatz und morpho-syntaktische Sprachleistungen im Vorschulalter. Sprache \& Kognition, 19(1/2), 15-21.

Grube, D., \& Seitz-Stein, K. (2012). Arbeitsgedächtnis und Rechnen. In M. Hasselhorn \& C. Zoelch (Hrsg.), Funktionsdiagnostik des Arbeitsgedächtnisses (S. 145-157). Göttingen: Hogrefe.

Halliday, M. A. K. (2004). An introduction to functional grammar (3. Aufl.). London: Arnold.

Hasselhorn, M., \& Gold, A. (2006). Pädagogische Psychologie. Erfolgreiches Lernen und Lehren. Stuttgart: Kohlhammer.

Heinze, A., Herwartz-Emden, L., \& Reiss, K. (2007). Mathematikkenntnisse und sprachliche Kompetenz bei Kindern mit Migrationshintergrund zu Beginn der Grundschulzeit. Zeitschrift für Pädagogik, 53(4), 562-581.

Heller, K.A., \& Perleth, C. (2000). Kognitiver Fähigkeitstest für 4. bis 12. Klassen: Revision (KFT 4-12+R). Göttingen: Beltz.

Hoeft, M., Wendt, H., \& Kasper, D. (2015). Familiäre Lebensumwelten in Europa - Zusammenhänge formeller und informeller häuslicher Aktivitäten zwischen Eltern und Kindern im Vorschulalter in den Förderdimensionen Early Literacy und Early Numeracy. In H. Wendt, T. C. Stubbe, K. Schwippert \& W. Bos (Hrsg.), 10 Jahre international vergleichende Schulleistungsforschung in der Grundschule: Vertiefende Analysen zu IGLU und TIMSS 2001 bis 2011 (S. 135-160). Münster: Waxmann.

Hoese, D. (2017). Kognitive Fähigkeiten und Mathematikleistungen im Grundschulalter: Kreuzverzögerte Effekte über vier Messzeitpunkte. Rostock: Universität Rostock. https://doi.org/10.18453/rosdok_ id00002521. 
Jansen, H., Mannhaupt, G., Marx, H., \& Skowronek, H. (2002). Bielefelder Screening zur Früherkennung von Lese-Rechtschreibschwierigkeiten (BISC). Göttingen: Hogrefe.

Kaufmann, L., \& von Aster, M. (2012). Diagnostik und Intervention bei Rechenstörung. Deutsches Ärzteblatt, 109(45), 767-778.

Kempert, S., Schalk, L., \& Saalbach, H. (2018). Sprache als Werkzeug des Lernens: Ein Überblick zu den kommunikativen und kognitiven Funktionen der Sprache und deren Bedeutung für den fachlichen Wissenserwerb. Psychologie in Erziehung und Unterricht. https://doi.org/10.2378/peu2018.art19d.

Kolkman, M. E., Kroesbergen, E. H., \& Leseman, P. P. M. (2014). Involvement of working memory in longitudinal development of number-magnitude skills. Infant and Child Development, 23(1), 36-50.

Krajewski, K. (2014). Förderung des Zahlverständnisses. In G. W. Lauth, M. Grünke \& J.C. Brunstein (Hrsg.), Interventionen bei Lernstörungen: Förderung, Training und Therapie in der Praxis (2. Aufl. S. 199-208). Göttingen: Hogrefe.

Krajewski, K., \& Ennemoser, M. (2010). Die Berücksichtigung begrenzter Arbeitsgedächtnisressourcen in Unterricht und Lernförderung. In H.-P. Trolldenier, W. Lenhard \& P. Marx (Hrsg.), Brennpunkte der Gedächtnisforschung. Entwicklungs- und pädagogisch-psychologische Perspektiven (S. 337-360). Göttingen: Hogrefe.

Krajewski, K., Küspert, P., \& Schneider, W. (2002). Deutscher Mathematiktest für erste Klassen (DEMAT 1+). Göttingen: Hogrefe.

Kyttälä, M., Aunio, P., Lepola, J., \& Hautamäki, J. (2013). The role of the working memory and language skills in the prediction of word problem solving in 4- to 7-year-old children. Educational Psychology, 34(6), 674-696.

Lang, F. R., Weiss, D., Stocker, A., \& von Rosenbladt, B. (2007). Assessing cognitive capacities in computer-assisted survey research: Two ultra-short tests of intellectual ability in the German socio-economic panel (SOEP). Journal of Applied Social Science Studies, 127, 183-192.

Lenhard, W., \& Schneider, W. (2006). ELFE 1-6 Ein Leseverständnistest für Erst- bis Sechstklässler. Göttingen: Hogrefe.

Lorenz, J.H. (2012). Kinder begreifen Mathematik: Frühe mathematische Bildung und Förderung. Entwicklung und Bildung in der Frühen Kindheit. Stuttgart: Kohlhammer.

Maier, H., \& Schweiger, F. (2008). Mathematik und Sprache. Zum Verstehen und Verwenden von Fachsprache im Mathematikunterricht. https://wwwmath.uni-muenster.de/reine/u/mollerh/data/ MaierSchweig11.pdf. Zugegriffen: 11. Dez. 2018.

Mannhaupt, G. (2006). Münsteraner Screening zur Früherkennung von Lese-Rechtschreibschwierigkeiten. Berlin: Cornelsen.

Melchers, P., \& Preuß, U. (2009). Kaufmann assessment battery for children (K-ABC) (8. Aufl.). Frankfurt: Pearson.

Muthén, B. O. \& Muthén, L. K. (2009). Mplus 5.21 [Computer Software]. Los Angeles, CA: Muthén \& Muthén.

Neumann, I., Duchhardt, C., Grüßing, M., Heinze, A., Knopp, E., \& Ehmke, T. (2013). Modeling and assessing mathematical competence over the lifespan. Journal for educational research online, 5 (2), S. 80-109. https://www.pedocs.de/volltexte/2013/8426/pdf/JERO_2013_2_Neumann_et_al_ Modeling_and_assessing_mathematical_competencies.pdf. Zugegriffen: 27. Nov. 2019.

Paetsch, J. (2016). Der Zusammenhang zwischen sprachlichen und mathematischen Kompetenzen bei Kindern deutscher und bei Kindern nicht-deutscher Familiensprache. Dissertation. Berlin: Freie Universität Berlin.

Prediger, S., Erath, K., \& Moser Opitz, E. (2019). The language dimension of mathematical difficulties. In A. Fritz, V. Haase \& P. Räsänen (Hrsg.), International Handbook of math learning difficulties: From the laboratory to the classroom (S. 437-455). Cham: Springer.

Röhm, A., Starke, A., \& Ritterfeld, U. (2017). Die Rolle von Arbeitsgedächtnis und Sprachkompetenz für den Erwerb mathematischer Basiskompetenzen im Vorschulalter. Psychologie in Erziehung und Unterricht, 64(2), 81-93. https://doi.org/10.2378/peu2016.art26d.

Schindler, V., Moser Opitz, E., Cadonau-Bieler, M., \& Ritterfeld, U. (2019). Überprüfung und Förderung des mathematischen Fachwortschatzes der Grundschulmathematik - eine empirische Studie. Journal für Mathematik-Didaktik, 40(1), 1-35. https://doi.org/10.1007/s13138-018-0135-2.

Schröder, A. \& Ritterfeld, U. (2014). Zur Bedeutung sprachlicher Barrieren im Mathematikunterricht der Primarstufe: Wissenschaftlicher Erkenntnisstand und Reflexion in der (Förder-)Schulpraxis. Forschung Sprache E-Journal für Sprachheilpädagogik, Sprachtherapie und Sprachförderung, 2, 49-69. https://www.forschung-sprache.eu/fileadmin/user_upload/Dateien/Heftausgaben/2014-1/FS_201401_49-69_Schroeder_Ritterfeld.pdf. Zugegriffen: 9. März 2020. 
Sfard, A., \& Kieran, C. (2008). Cognition as communication: rethinking learning-by-talking through multifaceted analysis of students' mathematical interactions. Mind, Culture, and Activitiy, 8(1), 42-76.

Sprachsensible Schulentwicklung (2017). Das Projekt „Sprachsensible Schulentwicklung“. Erfahrungen und Konzepte zur Umsetzung in Schulen. https://www.stiftung-mercator.de/media/downloads/ 3_Publikationen/2017/November/Das_Projekt_Sprachsensible_Schulentwicklung_Erfahrungen_ und_Konzepte_zur_Umsetzung_in_Schulen_Publikation_November_2017.pdf. Zugegriffen: 6. Juni 2019.

Tewes, U., Rossmann, P., \& Schallberger, U. (1999). Hamburg-Wechsler-Intelligenztest für Kinder III (HAWIK-III). Bern: Huber.

Tiedemann, K. (2017). Beschreibungen im Prozess. Eine Fallstudie zur fachbezogenen Sprachentwicklung im Kontext unterschiedlicher Darstellungen. In A. Neumann, M. Hagena, K. Schwippert \& D. Leiss (Hrsg.), Mathematik und Sprache. Empirischer Forschungsstand und unterrichtliche Herausforderungen (S. 63-68). Münster: Waxmann.

Weinert, S. (2010). Beziehungen zwischen Sprachentwicklung und Gedächtnisentwicklung. In H.-P. Trolldenier, W. Lenhard \& P. Marx (Hrsg.), Brennpunkte der Gedächtnisforschung: Entwicklungs- und pädagogisch-psychologische Perspektiven (S. 147-170). Göttingen: Hogrefe.

Wen, Z. (2012). Working memory and second language learning. International Journal of Applied Linguistics, 22(1), 1-22.

Zheng, X., Swanson, H.L., \& Marcoulides, G.A. (2011). Working memory components as predictors of children's mathematical word problem solving. Journal Experimental Child Psychology, (110), 481-498. https://doi.org/10.1016/j.jecp.2011.06.001 